\title{
Free vibration analysis of singly curved shells using the Isogeometric finite strip method
}

\author{
A. Borković ${ }^{1,2}$, G. Radenković ${ }^{1,3}$, D. Majstorović ${ }^{1}$, S. Milovanović ${ }^{1}$, D. Milašinović ${ }^{1,4}$, R. Cvijić ${ }^{1}$ \\ ${ }^{1}$ University of Banja Luka, Faculty of Architecture, Civil Engineering and Geodesy, Department of Mechanics \\ and Theory of Structures, 78000 Banja Luka, Bosnia and Herzegovina, aleksandar.borkovic@,aggf.unibl.org \\ ${ }^{2}$ Institute of Applied Mechanics, Graz University of Technology, Technikerstraße 4/II, 8010 Graz, Austria \\ ${ }^{3}$ Faculty of Civil Engineering, University of Belgrade, Bulevar kralja Aleksandra 73, 11000 Belgrade, Serbia \\ ${ }^{4}$ Faculty of Civil Engineering Subotica, University of Novi Sad, Kozaračka 2a, 24000 Subotica, Serbia
}

\begin{abstract}
:
A novel method for the spatial discretization of two-dimensional domains is derived and applied to the problem of free vibrations of singly curved shells. This new method utilizes a tensor product of two independent families of lines to discretize the geometry and kinematics of a surface. The first family consists of NURBS functions which are implemented in agreement with the isogeometric approach. The second family of lines is a carefully selected series which satisfies boundary conditions a priori. The present hybrid formulation unifies spatial discretization schemes of the semi-analytical Finite strip method and the Isogeometric analysis. The obtained method inherits many features of both of the underlying techniques, e.g., high continuity in both directions, decoupling of the governing equations, and exact initial geometry. Thorough numerical analysis shows that this novel method is well-suited for the efficient and accurate free vibration analysis of singly curved thin shells.
\end{abstract}

Keywords: Isogeometric analysis, Finite strip method, singly curved shells, thin-walled structures, free vibrations

\section{Introduction}

Due to their favorable structural properties, thin shells are extensively utilized in different areas of the contemporary engineering. In these applications thin shells are often exposed to various dynamic loads, which sometimes result in a violent structural response. Although many powerful design methods and tools exist, there is still a need within the industry for more accurate and efficient designs. These demands serve as the driving force for the development of novel numerical and analytical models for the dynamic analysis of thin shells.

The mechanical behavior of shells is well-researched area and several theories based on different approximations and assumptions have been proposed, [1]. A detailed review of the shell theories is beyond the scope of this article. For a comprehensive treatment of the vibrations of shells, references [2], [3], and [4] are recommended. In order to demonstrate the proposed method clearly, this study is restricted to the free vibrations of homogenous, isotropic, thin, cylindrical shells with uniform thickness. The classical theory of thin shells is readily based on the Kirchhoff-Love (KL) hypothesis which allows reduction from $3 \mathrm{D}$ to $2 \mathrm{D}$ while disregarding the transverse shear strains.

There are few classes of shells regarding the geometry of their midsurface, e.g., doubly and singly curved shells. The latter ones are often referred to as the cylindrical shells and are simpler to model. Amongst them, the circular cylindrical shells are the simplest to deal with due to their constant curvature. Another classification distinguishes between the open and closed shells. It is argued in [5] that the free vibration analysis of closed cylindrical shells is considerably more developed in comparison 
with the analysis of open shells. The authors point out that the assumed 2D displacement field for closed shells can be reduced to a quasi-1D problem via the Fourier decomposition of circumferential wave motion. On the contrary, there is no similar simplification for open shells.

The general method for the free vibration analysis of closed cylindrical shells with various boundary conditions is developed in [6] and [7] where the Flügge's governing equations of motion are utilized and the displacement components are approximated with the selected series. A similar computational model for the circular cylindrical shells is accomplished in [8] by adding the appropriate auxiliary functions to the Fourier series. The solution for this model is obtained by the requirement that the boundary-value problem is exactly satisfied at a finite number of points. The thin clamped-clamped circular cylinders are studied in [9] utilizing an approach that is analogous to the Rayleigh-Ritz method. The applied technique returns a sequence of explicit algebraic equations which does not require complex numerical procedures or the convergence analysis.

The exact solutions for open circular shells are derived in [10] by the separation of boundary conditions into primary and secondary ones. Results for the primary boundary conditions are obtained by the generalized Navier method while secondary and mixed boundary conditions are handled via superposition. The authors conclude that the solutions of four primary and nine secondary shells with elementary boundary conditions are sufficient to describe all the combinations of homogenous boundary conditions. Furthermore, the hybrid method which utilizes the finite element (FE) procedure and the classical shell theory is employed in the analysis of open cylindrical shells with simply supported edges, [11]. The displacement functions are derived from the Sanders' equation for thin cylindrical shells and high-precision solutions are obtained.

A formulation for the free vibration analysis of open cylindrical, conical, and spherical shells is developed in [5]. Displacement components are expanded as the Chebyshev polynomials of the first kind in both directions. The coefficients of these polynomials are considered as the generalized coordinates and the solution is found by the Rayleigh-Ritz procedure. Boundary conditions are introduced via artificial spring technique and excellent results are obtained. A similar treatment of boundary conditions is successfully applied in [12] for the free vibrations of open and closed shells. The authors applied spectro-geometric Rayleigh-Ritz method which approximates displacement with the Fourier cosine series and auxiliary functions. Moreover, the exact solutions for the free vibration of open and closed circular cylindrical shells based on the Donnell-Mushtari shell theory is derived in [13] using the separation of variables method. It has already been noted that cylindrical shells with an arbitrarily curved circumferential profile are more difficult to deal with. One approach for the free vibration analysis of these shells is considered in [14] utilizing the transfer matrix of the shell. Thin closed elliptic shells are analyzed in [15] using the spline-collocation method. Generally, for the shells with noncircular circumferential profile, some method of spatial discretization is required.

The standard Finite strip method (FSM) approximates the displacement field with a series of continuous functions in the longitudinal, and with the polynomials in the transverse direction, [16]. This procedure results in a set of finite strips connected at nodal lines, unlike the FE method which discretizes the domain into a set of elements connected at nodes, [17]. If the trigonometric series are employed, the formulation is considered as the semi-analytical method. For structures with regular geometries, it has several advantages over the FE method, [18], [19], [20], [21], [22]. There is another type of the method named the spline FSM. It utilizes spline functions instead of series, which improves the versatility of the method, [18]. The spline FSM is applied for the free vibration analysis of a singly curved shell panels in [23] where the Lagrange polynomials are used in the transverse, and B-splines are employed in the longitudinal direction. The doubly curved shells are considered in [24] with the restriction that the boundaries must align with the coordinate lines, as in the present formulation. Note that the buckling problems are tackled with the spline FSM as well, [25]. 
In this research, the non-uniform rational B-spline (NURBS) functions are utilized for the approximation along the one direction of the shell midsurface. These functions are implemented using the methodology of Isogeometric analysis (IGA), introduced in [26]. The basic idea of IGA is to utilize CAD basis functions in order to render a description of the geometry and kinematics. Many useful computational properties stem from this concept, such as the increased interelement continuity and the exact description of the initial geometry, [27], [28]. The problem of structural vibrations is investigated with IGA in [29] and [30], where it is shown that increased interelement continuity significantly improves the numerical discrete spectra. Furthermore, the first IGA KL shell element was developed in [31] where the authors argue that the method is ideally suited for KL shell modeling since the required $C^{1}$ continuity of the displacement field is easily achieved. This procedure allows for the development of the efficient KL shell models without the rotational degrees of freedom (DOF), [32]. Following the basic premises of IGA, several new computational models were developed, such as the isogeometric meshfree-coupling approach, [33], [34], and the isogeometric spline FSM, [35].

The aim of this research is to postulate a novel spatial discretization technique by blending the IGA and semi-analytical FSM, and to study its computational properties. An arbitrarily curved 1D plane geometry is employed following the isogeometric approach, similar as in [36]. This NURBS function is introduced in the standard semi-analytical FSM instead of Lagrange/Hermite polynomials. A similar concept is considered for the free vibration of cylindrical shells in [37]. The authors utilized quintic Bezier functions to represent the displacement field along the circumferential direction while the beam eigenfunctions are used in the longitudinal direction. Also, the effect of different continuities between the adjoining panels is discussed. Nevertheless, the present formulation is more general because it allows the investigation into the full set of $h p k$-refinement schemes which are the crucial part of IGA. This set is here expanded with the fourth mesh-improving technique, named t-refinement, which comprise straightforward increase in the number of series terms. Therefore, a novel hpkt-refinement space is considered for the first time. This developed approach is named the Isogeometric finite strip method (IGFSM) and it was conceived in [38] but without implementation. In order to validate the idea, the theory of doubly curved shells is briefly presented and the isogeometric finite strip for singly curved shell is derived as a special case. The numerical results show that all the main properties of IGA and FSM are preserved.

Concretely, the main advantages of the derived formulation are: (i) increased continuity along both directions, (ii) decoupling of system equations for some boundary conditions, (iii) exact description of initial geometry, and (iv) analytical integration of trigonometric functions along the longitudinal direction. On the other hand, the method is applicable for a narrow class of singly curved structures with constant properties along the longitudinal direction, which is its main shortcoming.

The paper is structured as follows. The next two sections deal with the geometry and kinematics of the doubly curved thin shell. Several classical assumptions are introduced in order to simplify the metric. In the fourth section, the equations of a singly curved shell are obtained as the special case and the isogeometric finite strip is derived. In addition, the trigonometric series which satisfy the boundary conditions are adopted and discussed. Numerical analysis of three characteristic examples of singly curved shells is given in the fifth section, and conclusions are given in the final section.

\section{Metric of the shell continuum}

A brief review of the metric of doubly curved shell is given in this section. The classical KL assumption is employed: straight line element is rigid and remains perpendicular to deformed shell midsurface. This assumption allows the reduction of a 3D continuum shell model into an arbitrarily shaped surface. The present analysis is conducted using the convective frame of reference while the complete shell kinematics is defined by the Cartesian components of translation of midsurface. 
The boldface lowercase and uppercase letters are used for vectors and tensors/matrices, respectively. The asterisk symbol designates a deformed configuration while the overbar indicates an equidistant surface. The quantities measured with respect to the local, curvilinear, coordinates are labeled with the caret symbol. The Greek index letters take values of 1 and 2 while the Latin indices take values of 1,2 , and 3 . The covariant and partial derivatives with respect to the $m^{\text {th }}$ coordinate are designated with ( ) $m$ and ( ),m, respectively.

Details of the NURBS-based IGA geometry modeling are excluded since the comprehensive treatments of this subject are readily available, [26], [31], [39].

\subsection{Metric of the midsurface}

The midsurface of a shell with respect to the Cartesian coordinates is determined by its position vector $\mathbf{r}=\left\{x=x^{l}, y=x^{2}, z=x^{3}\right\}$, Fig. 1. Let us define this vector as a tensor product of two independent families of lines:

$$
\mathbf{r}=\mathbf{r}(\xi, \eta)=x^{k} \mathbf{i}_{k}=\sum_{I=1}^{N} R_{I}(\xi) \sum_{J=1}^{M} F_{J}(\eta) \mathbf{r}_{I J}, \quad x^{k}=\sum_{I=1}^{N} R_{I}(\xi) \sum_{J=1}^{M} f_{J}^{k}(\eta) x_{I J}^{k}, \quad \mathbf{r}_{I J}=x_{I J}^{k} \mathbf{i}_{k},
$$

where $R_{I}(\xi)$ is NURBS basis function of $I^{\text {th }}$ control point, while $F_{J}(\eta)$ is $J^{\text {th }}$ term of a series:

$$
F_{J}=\left\{f_{J}^{1}, f_{J}^{2}, f_{J}^{3}\right\}
$$

Note that different series $f_{J}^{k}$ are allowed for the distribution of each Cartesian component. $\mathbf{r}_{I J}$ is the position vector of the $I^{\text {th }}$ control point for the $J^{\text {th }}$ series term. $N$ is the total number of control points along the $\xi$ direction while $M$ is the total number of series terms used for the description of geometry. Furthermore, $\mathbf{i}_{\mathrm{m}}$ are the base vectors of the Cartesian coordinate system while $\theta^{1}=\xi$ and $\theta^{2}=\eta$ are local curvilinear coordinates, see Fig. 1.

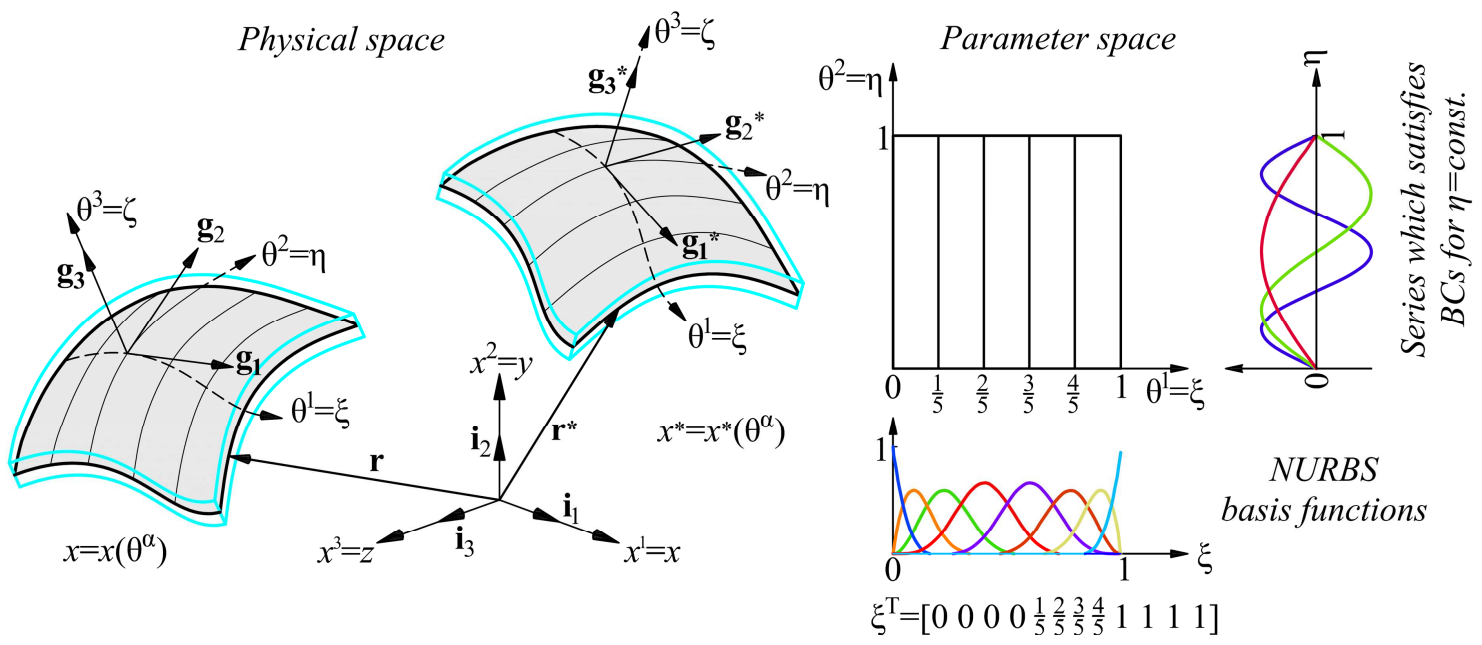

Fig. 1. The mapping between the reference and deformed configurations in physical space (left), and parameter space (right). An example of general basis functions is shown next to the parameter space.

Tangent vectors of the midsurface are defined as:

$$
\mathbf{g}_{\alpha}=\frac{\partial \mathbf{r}}{\partial \theta^{\alpha}}=x_{, \alpha}^{m} \mathbf{i}_{m}
$$

where $x_{, \alpha}^{m}$ are the partial derivatives of the Cartesian components of the midsurface with respect to $\theta^{\alpha}$ coordinates. For the adopted description of geometry, these vectors are: 


$$
\begin{aligned}
& \mathbf{g}_{1}=\frac{\partial \mathbf{r}}{\partial \xi}=x_{, 1}^{k} \mathbf{i}_{k}=\sum_{I=1}^{N} R_{I, 1}(\xi) \sum_{J=1}^{M} F_{J}(\eta) \mathbf{r}_{I J}, \mathbf{g}_{2}=\frac{\partial \mathbf{r}}{\partial \eta}=x_{, 2}^{k} \mathbf{i}_{k}=\sum_{I=1}^{N} R_{I}(\xi) \sum_{J=1}^{M} F_{J, 2}(\eta) \mathbf{r}_{I J}, \\
& x_{, \alpha}^{k}=\sum_{I=1}^{N} \sum_{J=1}^{M}\left[\delta_{\alpha}^{1} R_{I, 1}(\xi) f_{J}^{k}(\eta)+\delta_{\alpha}^{2} R_{I}(\xi) f_{J, 2}^{k}(\eta)\right] x_{I J}^{k}
\end{aligned}
$$

where $\delta_{\alpha}^{\beta}$ is the Kronecker delta. The third coordinate $\theta^{3}=\zeta$ is straight and perpendicular to the $\theta^{\alpha}$ coordinate lines. Therefore, the base vector $\mathbf{g}_{3}$ and its reciprocal counterpart $\mathbf{g}^{3}$ are the same, have the unit length, and represent the normal of midsurface:

$$
\mathbf{g}_{3}=\mathbf{g}^{3}=\mathbf{n}=\frac{1}{\sqrt{g}}\left(\mathbf{g}_{1} \times \mathbf{g}_{2}\right)=x_{, 3}^{m} \mathbf{i}_{m}, x_{, 3}^{m}=x_{m, 3}=\frac{1}{\sqrt{g}} x_{1,2}^{k} x_{, 2}^{l} e_{k l m},
$$

where $e_{m n k}$ is the permutation symbol while $g$ is the determinant of metric tensor of midsurface:

$$
g_{i j}=x_{, i}^{k} x_{k, j}=\left[\begin{array}{ccc}
x_{, 1}^{m} x_{m, 1} & x_{, 1}^{m} x_{m, 2} & 0 \\
x_{, 2}^{m} x_{m, 1} & x_{, 2}^{m} x_{m, 2} & 0 \\
0 & 0 & 1
\end{array}\right]=\left[\begin{array}{ccc}
g_{11} & g_{12} & 0 \\
g_{21} & g_{22} & 0 \\
0 & 0 & 1
\end{array}\right], g_{12}=g_{21}, g=\operatorname{det} g_{i j}=g_{11} g_{22}-g_{12}^{2} .
$$

The reciprocal metric tensor of midsurface is:

$$
g^{i j}=g_{i j}^{-1}=\frac{1}{g}\left[\begin{array}{ccc}
g_{22} & -g_{12} & 0 \\
-g_{12} & g_{11} & 0 \\
0 & 0 & g
\end{array}\right]=\left[\begin{array}{ccc}
g^{11} & g^{12} & 0 \\
g^{21} & g^{22} & 0 \\
0 & 0 & 1
\end{array}\right], \quad \operatorname{det} g^{i j}=1 / g,
$$

and the reciprocal tangent vectors are:

$$
\begin{aligned}
& \mathbf{g}^{1}=\frac{1}{\sqrt{g}}\left(\mathbf{g}_{2} \times \mathbf{g}_{3}\right)=x_{n}^{1} \mathbf{i}^{n}, \quad x_{n}^{1}=\frac{1}{\sqrt{g}} x_{, 2}^{m} x_{, 3}^{k} e_{m k n}, \\
& \mathbf{g}^{2}=\frac{1}{\sqrt{g}}\left(\mathbf{g}_{3} \times \mathbf{g}_{1}\right)=x_{n}^{2} \mathbf{i}^{n}, x_{n}^{2}=\frac{1}{\sqrt{g}} x_{, 3}^{m} x_{, 1}^{k} e_{m k n} .
\end{aligned}
$$

Furthermore, the derivatives of base vectors are calculated as:

$$
\mathbf{g}_{\alpha, \beta}=x_{, \alpha \beta}^{m} \mathbf{i}_{m}=\left\{\begin{array}{l}
x_{, \alpha \beta}^{m} x_{m, k} \mathbf{g}^{k}=\Gamma_{\alpha \beta k} \mathbf{g}^{k} \\
x_{, \alpha \beta}^{m} x_{m}^{n} \mathbf{g}_{n}=\Gamma_{\alpha \beta}^{n} \mathbf{g}_{n}
\end{array} \Rightarrow \Gamma_{\alpha \beta}^{n}=g^{n k} \Gamma_{\alpha \beta k},\right.
$$

where $\Gamma_{\alpha \beta k}$ and $\Gamma_{\alpha \beta}^{n}$ are the Christoffel symbols of the first and the second kind, respectively. For the introduced tensor product, Eq. (1), these derivatives are, [38]:

$$
\mathbf{g}_{\alpha, \beta}=\sum_{I=1}^{N}\left[\delta_{\alpha}^{1} \delta_{\beta}^{1} R_{I, 11}(\xi) \sum_{J=1}^{M} F_{J}(\eta) \mathbf{r}_{I J}+\delta_{\alpha}^{2} \delta_{\beta}^{2} R_{I}(\xi) \sum_{J=1}^{M} F_{J, 22}(\eta) \mathbf{r}_{I J}+\left(\delta_{\alpha}^{1} \delta_{\beta}^{2}+\delta_{\alpha}^{2} \delta_{\beta}^{1}\right) R_{I, 1}(\xi) \sum_{J=1}^{M} F_{J, 2}(\eta) \mathbf{r}_{I J}\right] .
$$

The Christoffel symbols $\Gamma_{\alpha \beta}^{3}$ represent the components of the curvature tensor and we will designate them as $\Gamma_{\alpha \beta}^{3}=b_{\alpha \beta}$ :

$$
\mathbf{g}_{3, \alpha}=-\Gamma_{3 \alpha}^{\mu} \mathbf{g}_{\mu}=-b_{\alpha}^{\mu} \mathbf{g}_{\mu}, b_{\alpha}^{\mu}=g^{\mu v} b_{v \alpha},
$$

where $b_{\alpha}^{\mu}$ and $b_{v \alpha}$ are the mixed and covariant components of the curvature tensor, respectively, [1].

\subsection{Metric of an equidistant surface}

The tangent vectors of an equidistant surface are the derivatives of its position vector: 


$$
\begin{aligned}
& \overline{\mathbf{r}}=\mathbf{r}+\zeta \mathbf{g}_{3}, \\
& \overline{\mathbf{g}}_{\alpha}=\frac{\partial \overline{\mathbf{r}}}{\partial \theta^{\alpha}}=\mathbf{g}_{\alpha}-\zeta b_{\alpha}^{v} \mathbf{g}_{v}=\left(\delta_{\alpha}^{v}-\zeta b_{\alpha}^{v}\right) \mathbf{g}_{v}=\bar{C}_{\alpha}^{v} \mathbf{g}_{v}, \bar{C}_{\alpha}^{v}=\delta_{\alpha}^{v}-\zeta b_{\alpha}^{v},
\end{aligned}
$$

where $\bar{C}_{\alpha}^{v}$ are components of the shift tensor, [40]. The covariant components of the metric tensor at an equidistant surface are then:

$$
\bar{g}_{\alpha \beta}=\overline{\mathbf{g}}_{\alpha} \cdot \overline{\mathbf{g}}_{\beta}=\bar{C}_{\alpha}^{\mu} \bar{C}_{\beta}^{v} g_{\mu v}=\left(\delta_{\alpha}^{\mu}-\zeta b_{\alpha}^{\mu}\right)\left(\delta_{\beta}^{v}-\zeta b_{\beta}^{v}\right) g_{\mu \nu}=g_{\alpha \beta}-2 \zeta b_{\alpha \beta}+\zeta^{2} b_{\alpha}^{\mu} b_{\beta}^{v} g_{\mu \nu} .
$$

For thin and moderately thick shells, these components are often approximated by neglecting the quadratic term with respect to $\zeta$ coordinate, [41]:

$$
\bar{g}_{\alpha \beta} \approx g_{\alpha \beta}-2 \zeta b_{\alpha \beta} .
$$

The reciprocal metric tensor at an equidistant surface can be calculated by the inversion of the previous expression. The subject of this research are shells with small to medium curviness, i.e., $K h<0.1$, where $K=b_{1}^{1}+b_{2}^{2}$ is the maximum value of the trace of the curvature tensor. For these shells, another restrictive assumption can be posed:

$$
\bar{g}^{\alpha \beta}=\overline{\mathbf{g}}^{\alpha} \overline{\mathbf{g}}^{\beta} \approx \mathbf{g}^{\alpha} \mathbf{g}^{\beta}=g^{\alpha \beta},
$$

which allows the simple representation of a constitutive relation at an equidistant surface, [40]. A close inspection of the Eqs. (14) and (15) suggests that the covariant metric at an equidistant surface is more accurate than its contravariant counterpart, although both are estimated here.

Finally, let us approximate the differential volume of the shell as:

$$
\mathrm{d} v=\left(\mathrm{d} \xi \mathbf{g}_{1} \times \mathrm{d} \eta \mathbf{g}_{2}\right) \cdot \mathrm{d} \zeta \mathbf{g}_{3} \approx \sqrt{g} \mathrm{~d} \xi \mathrm{d} \eta d \zeta=\mathrm{d} a \mathrm{~d} \zeta,
$$

where $\mathrm{d} a=\sqrt{g} \mathrm{~d} \xi \mathrm{d} \eta$ is the differential area of midsurface.

\section{Kirchhoff-Love shell theory}

For the convective coordinate frame, the Lagrange strain is the difference between the current and reference metrics:

$$
\varepsilon_{\alpha \beta}=\frac{1}{2}\left(g_{\alpha \beta}^{*}-g_{\alpha \beta}\right)
$$

Due to the KL hypothesis, the components of the shear strain along the normal direction vanish, i.e. $\varepsilon_{\alpha 3}=0$, and the position of a line element is completely determined by the translation of midsurface. This fact gave impetus for the development of so-called rotation-free shell theories, [31].

\subsection{Kinematics of the shell}

The position vector of deformed midsurface is:

$$
\mathbf{r}^{*}=\mathbf{r}+\mathbf{u},
$$

where the displacement $\mathbf{u}$ is approximated with the tensor product of the NURBS and selected series which satisfies boundary conditions for $\eta=$ const. Let us assume that this series is the same as that utilized for the description of the geometry, Eq. (1):

$$
\mathbf{u}=u^{m} \mathbf{i}_{m}=\sum_{I=1}^{N} R_{I}(\xi) \sum_{J=1}^{M} F_{J}(\eta) \mathbf{u}_{I J}, \quad u^{m}=\sum_{I=1}^{N} R_{I}(\xi) \sum_{J=1}^{M} f_{J}^{m}(\eta) u_{I J}^{m} .
$$


$\mathbf{u}_{I J}$ is the vector which consists of the Cartesian displacement components of the $I^{\text {th }}$ control point for the $J^{t h}$ series term. In order to have a seamless transition to the discrete equation of motion which follows in Section 4, let us introduce the matrix of basis functions $\mathbf{N}$ such that the Eq. (19) can be written as:

$$
\mathbf{u}=\mathbf{N q}
$$

where:

$$
\begin{aligned}
& \mathbf{q}^{\mathrm{T}}=\left[\begin{array}{llllll}
\mathbf{u}_{11}^{\mathrm{T}} & \mathbf{u}_{12}^{\mathrm{T}} & \ldots & \mathbf{u}_{I J}^{\mathrm{T}} & \ldots & \mathbf{u}_{N M}^{\mathrm{T}}
\end{array}\right], \quad \mathbf{u}_{I J}^{\mathrm{T}}=\left[\begin{array}{lll}
u_{I J}^{1} & u_{I J}^{2} & u_{I J}^{3}
\end{array}\right], \\
& \mathbf{N}=\left[\begin{array}{llllll}
\mathbf{N}_{11} & \mathbf{N}_{12} & \ldots & \mathbf{N}_{I J} & \ldots & \mathbf{N}_{N M}
\end{array}\right], \\
& \mathbf{N}_{I J}=\left[\begin{array}{ccc}
N_{I J}^{1} & 0 & 0 \\
0 & N_{I J}^{2} & 0 \\
0 & 0 & N_{I J}^{3}
\end{array}\right], \quad N_{I J}^{m}=R_{I} f_{j}^{m} .
\end{aligned}
$$

The acceleration field is obtained as the second material derivative of the displacement field:

$$
\ddot{\mathbf{u}}=\ddot{u}^{n} \mathbf{i}_{n},
$$

and it is represented analogously:

$$
\begin{aligned}
& \ddot{\mathbf{u}}=\mathbf{N} \ddot{\mathbf{q}}, \\
& \ddot{\mathbf{q}}^{\mathrm{T}}=\left[\begin{array}{llllll}
\ddot{\mathbf{u}}_{11}^{\mathrm{T}} & \ddot{\mathbf{u}}_{12}^{\mathrm{T}} & \ldots & \ddot{\mathbf{u}}_{I J}^{\mathrm{T}} & \ldots & \ddot{\mathbf{u}}_{N M}^{\mathrm{T}}
\end{array}\right], \quad \ddot{\mathbf{u}}_{I J}=\left[\begin{array}{llll}
\ddot{u}_{I J}^{1} & \ddot{u}_{I J}^{2} & \ddot{u}_{I J}^{3}
\end{array}\right] .
\end{aligned}
$$

The strain tensor of the midsurface, neglecting the nonlinear contributions, is:

$$
\begin{aligned}
& \left.\varepsilon_{\alpha \beta}=\frac{1}{2}\left(g_{\alpha \beta}^{*}-g_{\alpha \beta}\right)=\frac{1}{2}\left(\mathbf{g}_{\alpha}^{*} \cdot \mathbf{g}_{\beta}^{*}-\mathbf{g}_{\alpha} \cdot \mathbf{g}_{\beta}\right)=\frac{1}{2}\left[\left(\mathbf{g}_{\alpha}+\mathbf{u}_{\mid \alpha}\right) \cdot\left(\mathbf{g}_{\beta}+\mathbf{u}_{\mid \beta}\right)-\mathbf{g}_{\alpha} \cdot \mathbf{g}_{\beta}\right)\right]= \\
& \frac{1}{2}\left(\mathbf{g}_{\alpha} \cdot \mathbf{u}_{\mid \beta}+\mathbf{g}_{\beta} \cdot \mathbf{u}_{\mid \alpha}+\mathbf{u}_{\mid \alpha} \cdot \mathbf{u}_{\mid \beta}\right) \approx \frac{1}{2}\left(\mathbf{g}_{\alpha} \cdot \mathbf{u}_{\mid \beta}+\mathbf{g}_{\beta} \cdot \mathbf{u}_{\mid \alpha}\right)=\frac{1}{2}\left(x_{, \alpha}^{k} \hat{u}_{k \mid \beta}+x_{, \beta}^{k} \hat{u}_{k \mid \alpha}\right),
\end{aligned}
$$

where $\hat{u}_{m}$ are the components of the displacement vector with respect to the local curvilinear coordinates:

$$
\mathbf{u}=\hat{u}_{m} \mathbf{g}^{m}=u^{k} \mathbf{i}_{k} \Rightarrow \hat{u}_{m}=u_{k} \mathbf{i}^{k} \cdot \mathbf{g}_{m}=x_{, m}^{k} u_{k} .
$$

Now, the covariant components of the infinitesimal strain with respect to the Cartesian basis can be written as:

$$
\varepsilon_{\alpha \beta}=\frac{1}{2}\left(x_{, \alpha}^{k} u_{k, \beta}+x_{, \beta}^{k} u_{k, \alpha}+2 x_{, \alpha \beta}^{k} u_{k}-2 \Gamma_{\alpha \beta}^{m} x_{, m}^{n} u_{n}\right)=\frac{1}{2}\left(x_{, \alpha}^{k} u_{k, \beta}+x_{, \beta}^{k} u_{k, \alpha}\right)=\frac{1}{2}\left(\mathbf{g}_{\alpha} \cdot \mathbf{u}_{, \beta}+\mathbf{g}_{\beta} \cdot \mathbf{u}_{, \alpha}\right) .
$$

\subsection{Strain at an equidistant surface}

The representation of a strain at each point of a structure via reference quantities allows a dimensional reduction from $3 \mathrm{D}$ to $2 \mathrm{D}$. Using assumption (14), the strain at an equidistant surface reduces to:

$$
\bar{\varepsilon}_{\alpha \beta}=\varepsilon_{\alpha \beta}(\zeta)=\frac{1}{2}\left(\bar{g}_{\alpha \beta}^{*}-\bar{g}_{\alpha \beta}\right) \approx \frac{1}{2}\left(g_{\alpha \beta}^{*}-g_{\alpha \beta}\right)-\zeta\left(b_{\alpha \beta}^{*}-b_{\alpha \beta}\right)=\varepsilon_{\alpha \beta}-\zeta \kappa_{\alpha \beta},
$$

where $\kappa_{\alpha \beta}=b_{\alpha \beta}^{*}-b_{\alpha \beta}$ is the tensor of the curvature change of midsurface. If we introduce the expression for the displacement gradient with respect to $\zeta$ coordinate, [1]:

$$
\mathbf{u}_{, 3}=-x_{m, 3} u_{, \alpha}^{m} x_{k}^{\alpha} \mathbf{i}^{k}=-\left(\mathbf{g}_{3} \cdot \mathbf{u}_{, \alpha}\right) \mathbf{g}^{\alpha},
$$

the curvature change can be calculated as: 


$$
\begin{aligned}
\kappa_{\alpha \beta}= & b_{\alpha \beta}^{*}-b_{\alpha \beta}=\mathbf{g}_{\alpha, \beta}^{*} \cdot \mathbf{g}_{3}^{*}-\mathbf{g}_{\alpha, \beta} \cdot \mathbf{g}_{3}=\left(\mathbf{g}_{\alpha}+\mathbf{u}_{, \alpha}\right)_{, \beta} \cdot\left(\mathbf{g}_{3}+\mathbf{u}_{, 3}\right)-\mathbf{g}_{\alpha, \beta} \cdot \mathbf{g}_{3} \approx \\
& \mathbf{u}_{, \alpha \beta} \cdot \mathbf{g}_{3}+\mathbf{g}_{\alpha, \beta} \cdot \mathbf{u}_{, 3}=\mathbf{u}_{, \alpha \beta} \cdot \mathbf{g}_{3}-\mathbf{g}_{\alpha, \beta} \cdot\left(\mathbf{g}_{3} \cdot \mathbf{u}_{, \mu}\right) \mathbf{g}^{\mu}= \\
& \mathbf{u}_{, \alpha \beta} \cdot \mathbf{g}_{3}-\Gamma_{\alpha \beta}^{k} \mathbf{g}_{k} \cdot\left(\mathbf{g}_{3} \cdot \mathbf{u}_{, \mu}\right) \mathbf{g}^{\mu}=\mathbf{g}_{3} \cdot\left(\mathbf{u}_{, \alpha \beta}-\Gamma_{\alpha \beta}^{\mu} \mathbf{u}_{, \mu}\right)=x_{, 3}^{k}\left(u_{k, \alpha \beta}-\Gamma_{\alpha \beta}^{\mu} u_{k, \mu}\right) .
\end{aligned}
$$

The equations (26), (27), and (29) define the relations between the equidistant and reference strains of a KL shell within the scope of the linear theory. The formulation of the isogeometric finite strip for the structural analysis of a doubly curved KL shell is based on them.

\section{Isogeometric finite strip formulation for a singly curved shell}

In this section, we will limit our considerations to singly curved thin shells. For these structures, components of the curvature tensor at initial configuration are, Fig. 2:

$$
b_{1}^{1} \neq 0, \quad b_{2}^{2}=b_{2}^{1}=b_{1}^{2}=0,
$$

and the series used for the approximation of reference geometry, Eq. (2), reduce to the classic ones used in the semi-analytical FSM, [21]:

$$
F_{J}=\left\{f_{J}^{1}, f_{J}^{2}, f_{J}^{3}\right\}=\{1, \eta, 1\} .
$$

Due to this restriction, the introduced hybrid method can be viewed as a partial IGFSM. It is not complete since the same basis functions are not used for the geometry and kinematics. Concretely, the NURBS is utilized for both, but different series are employed for the kinematics and geometry here. For a more general setup, this approach can be improved by adding the auxiliary functions to some adopted trigonometric series and setting up the appropriate coefficients to zero, [21].

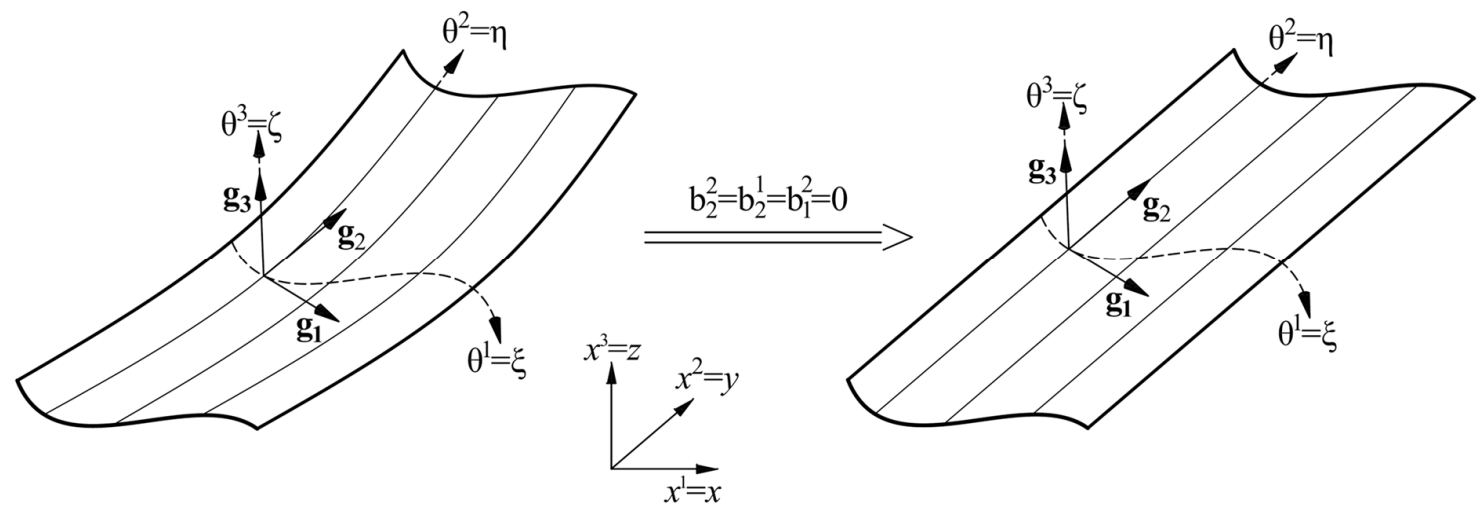

Fig. 2. Reduction of a doubly to a singly curved shell. An isogeometric finite strip model.

For the singly curved shells, the base vectors of the midsurface are simplified:

$$
\mathbf{g}_{1}=\left\{x_{, 1}^{1}, 0, x_{, 1}^{3}\right\}, \mathbf{g}_{2}=\{0,1,0\}, \mathbf{g}_{3}=\left\{x_{, 3}^{1}, 0, x_{, 3}^{3}\right\},
$$

as well as the components of metric tensors:

$$
\begin{aligned}
& g_{11}=\mathbf{g}_{1} \cdot \mathbf{g}_{1}, \quad g_{12}=g_{21}=0, \quad g_{22}=1 \text {, } \\
& g^{11}=1 / g_{11}, g^{12}=g^{21}=0, g^{22}=1 \text {. }
\end{aligned}
$$

Consequently, the expressions for the reference strains of the midsurface, Eqs. (26) and (29), reduce to: 


$$
\begin{aligned}
& \varepsilon_{11}=x_{k, 1} u_{, 1}^{k}=x_{1,1} u_{, 1}^{1}+x_{3,1} u_{, 1}^{3}, \\
& \varepsilon_{22}=x_{k, 2} u_{, 2}^{k}=u_{, 2}^{2}, \\
& 2 \varepsilon_{12}=x_{k, 2} u_{, 1}^{k}+x_{k, 1} u_{, 2}^{k}=u_{, 1}^{2}+x_{1,1} u_{, 2}^{1}+x_{3,1} u_{, 2}^{3}, \\
& \kappa_{11}=x_{k, 3}\left(u_{, 11}^{k}-\Gamma_{11}^{\mu} u_{, \mu}^{k}\right)=x_{1,3} u_{, 11}^{1}+x_{3,3} u_{, 11}^{3}-\Gamma_{11}^{1}\left(x_{1,3} u_{, 1}^{1}+x_{3,3} u_{, 1}^{3}\right), \\
& \kappa_{22}=x_{k, 3}\left(u_{, 22}^{k}-\Gamma_{22}^{\mu} u_{, \mu}^{k}\right)=x_{1,3} u_{, 22}^{1}+x_{3,3} u_{, 22}^{3}, \\
& 2 \kappa_{12}=2 x_{k, 3}\left(u_{, 12}^{k}-\Gamma_{12}^{\mu} u_{, \mu}^{k}\right)=2\left(x_{1,3} u_{, 12}^{1}+x_{3,3} u_{, 12}^{3}\right) .
\end{aligned}
$$

In line with the previous derivation, we will develop the isogeometric finite strip of a singly curved KL shell using the principle of virtual work. The generalized coordinates are the displacement components of control points for each series term.

The classic Saint Venant-Kirchhoff material model is applied. If the plane stress and plane strain conditions are enforced, the stress-strain relation reduces to:

$$
\sigma^{\alpha \beta}=2 \mu\left(g^{\alpha v} g^{\beta \gamma}+\frac{v}{1-v} g^{\alpha \beta} g^{v \gamma}\right) \varepsilon_{v \gamma}=D^{\alpha \beta v \gamma} \varepsilon_{v \gamma},
$$

where $D^{\alpha \beta v}$ are the components of the constitutive tensor while $\mu$ and $v$ are Lamé constants.

\subsection{Principle of virtual work}

The principle of virtual work states that at any configuration, the total work of external, internal, and inertial forces is zero for any admissible virtual state of motion. If the external effects are neglected, it can be written as:

$$
\delta W=\int_{v} \bar{\sigma}^{i j} \delta \bar{\varepsilon}_{i j} d v+\int_{v} \rho \ddot{\bar{u}}^{i} \delta \bar{u}_{i} d v=\int_{v} \overline{\boldsymbol{\sigma}}: \delta \overline{\boldsymbol{\varepsilon}} d v+\int_{v} \rho \ddot{\overline{\mathbf{u}}} \cdot \delta \overline{\mathbf{u}} d v=0,
$$

where $\sigma$ is the Cauchy stress tensor, $\varepsilon$ is the strain tensor, and $\ddot{\mathbf{u}}$ is the vector of accelerations, all of which are observed at an equidistant surface.

In order to reduce the computational model from $3 \mathrm{D}$ to $2 \mathrm{D}$, it is necessary to integrate Eq. (36) along the thickness. The internal term reduces to:

$$
\int_{v} \bar{\sigma}^{\alpha \beta} \delta \bar{\varepsilon}_{\alpha \beta} \mathrm{d} v=\int_{a}\left(N^{\mu v} \delta \varepsilon_{\mu v}+M^{\mu v} \delta \kappa_{\mu v}\right) \mathrm{d} a,
$$

where $N^{\mu v}$ and $M^{\mu v}$ are the stress resultants and stress couples which are energetically conjugated with the reference strains of the midsurface, $\varepsilon_{\mu \nu}$ and $\kappa_{\mu \nu}$ :

$$
N^{\alpha \beta}=\int_{-h / 2}^{h / 2} \bar{\sigma}^{\alpha \beta} \mathrm{d} \zeta, M^{\alpha \beta}=-\frac{1}{2} \int_{-h / 2}^{h / 2} \zeta \bar{\sigma}^{\alpha \beta} \mathrm{d} \zeta .
$$

If we assume that the effect of rotational inertia is negligible and that the material is homogenous, inertial term of virtual work reduces to:

$$
\int_{v} \rho \ddot{\bar{u}}^{i} \delta \bar{u}_{i} d v=\rho h \int_{a} \ddot{u}^{i} \delta u_{i} d a
$$

By introducing the vectors of generalized section forces and reference strains of the shell midsurface:

$$
\begin{aligned}
\mathbf{f}^{\mathrm{T}} & =\left[\begin{array}{llllll}
N^{11} & N^{22} & N^{12} & M^{11} & M^{22} & M^{12}
\end{array}\right], \\
\mathbf{e}^{\mathrm{T}} & =\left[\begin{array}{lllllll}
\varepsilon_{11} & \varepsilon_{22} & 2 \varepsilon_{12} & \kappa_{11} & \kappa_{22} & 2 \kappa_{12}
\end{array}\right],
\end{aligned}
$$


the Eq. (36) can be written in matrix form as:

$$
\delta W=\int_{a} \mathbf{f}^{\mathrm{T}} \delta \mathbf{e} \mathrm{d} a+\rho h \int_{a} \ddot{\mathbf{u}}^{\mathrm{T}} \delta \mathbf{u} \mathrm{d} a=0 .
$$

\subsection{Stress-strain relation}

The integration represented with the Eqs. (37) and (38) is the crucial step for the derivation of structural elements, since it allows a calculation of the complete virtual work by the reference quantities of the midsurface. For this, the constitutive relation between the stress and strain at an arbitrary point of the shell continuum is required. Due to the assumption (15), the stress-strain relation is here simplified:

$$
\bar{\sigma}^{\alpha \beta}=2 \mu\left(\bar{g}^{\alpha v} \bar{g}^{\beta \gamma}+\frac{v}{1-v} \bar{g}^{\alpha \beta} \bar{g}^{v \gamma}\right) \bar{\varepsilon}_{v \gamma} \approx 2 \mu\left(g^{\alpha v} g^{\beta \gamma}+\frac{v}{1-v} g^{\alpha \beta} g^{v \gamma}\right) \bar{\varepsilon}_{v \gamma}=D^{\alpha \beta v \gamma} \bar{\varepsilon}_{v \gamma} .
$$

In this way, the constitutive relations between the energetically conjugated section forces and reference strains are significantly reduced:

$$
\begin{gathered}
N^{\alpha \beta}=D_{M}^{\alpha \beta \gamma \lambda} \varepsilon_{\gamma \lambda}+D_{M B}^{\alpha \beta \gamma \lambda} \kappa_{\gamma \lambda}=h D^{\alpha \beta \gamma \lambda} \varepsilon_{\gamma \lambda}, \\
M^{\alpha \beta}=D_{B M}^{\alpha \beta \gamma \lambda} \varepsilon_{\gamma \lambda}+D_{B}^{\alpha \beta \gamma \lambda} \kappa_{\gamma \lambda}=\frac{h^{3}}{12} D^{\alpha \beta \gamma \lambda} \kappa_{\gamma \lambda},
\end{gathered}
$$

because:

$$
\begin{aligned}
& D_{M}^{\alpha \beta \gamma \lambda}=\int_{-h / 2}^{h / 2} D^{\mu \nu \delta \varphi} \mathrm{d} \zeta=h D^{\mu \nu \delta \varphi}, \\
& D_{M B}^{\alpha \beta \gamma \lambda}=-\int_{-h / 2}^{h / 2} \zeta D^{\mu \nu \delta \phi} \mathrm{d} \zeta=0, \\
& D_{B M}^{\alpha \beta \gamma \lambda}=-\int_{-h / 2}^{h / 2} \zeta D^{\mu \nu \delta \phi} \mathrm{d} \zeta=D_{M B}^{\gamma \lambda \beta}=0, \\
& D_{B}^{\alpha \beta \gamma \lambda}=\int_{-h / 2}^{h / 2} \zeta^{2} D^{\mu \nu \delta \varphi} \mathrm{d} \zeta=\frac{h^{3}}{12} D^{\mu \nu \delta \varphi} .
\end{aligned}
$$

Now, the Eq. (43) can be represented in a compact form as:

$$
\mathbf{f}=\mathbf{D e}
$$

where $\mathbf{D}$ is the constitutive tensor:

$$
\mathbf{D}=\left[\begin{array}{cc}
\mathbf{D}_{M} & \mathbf{0} \\
\mathbf{0} & \mathbf{D}_{B}
\end{array}\right]=\left[\begin{array}{cc}
D_{M}^{\alpha \beta \gamma \lambda} & 0 \\
0 & D_{B}^{\alpha \beta \gamma \lambda}
\end{array}\right] \mathbf{g}_{\alpha} \otimes \mathbf{g}_{\beta} \otimes \mathbf{g}_{\gamma} \otimes \mathbf{g}_{\lambda} .
$$

\subsection{Discrete equation of motion}

Since we are dealing with linearized theory, all quantities are measured with respect to the initial configuration. Therefore, the variations of reference strains are simply calculated by:

$$
\begin{aligned}
& \delta \varepsilon_{\alpha \beta}=\delta \frac{1}{2}\left(\mathbf{g}_{\alpha} \cdot \mathbf{u}_{, \beta}+\mathbf{g}_{\beta} \cdot \mathbf{u}_{, \alpha}\right)=\frac{1}{2}\left(\mathbf{g}_{\alpha} \cdot \delta \mathbf{u}_{, \beta}+\mathbf{g}_{\beta} \cdot \delta \mathbf{u}_{, \alpha}\right), \\
& \delta \kappa_{\alpha \beta}=\delta\left[\mathbf{g}_{3} \cdot\left(\mathbf{u}_{\alpha, \beta}-\Gamma_{\alpha \beta}^{\mu} \mathbf{u}_{\mu}\right)\right]=\mathbf{g}_{3} \cdot\left(\delta \mathbf{u}_{\alpha, \beta}-\Gamma_{\alpha \beta}^{\mu} \delta \mathbf{u}_{\mu}\right) .
\end{aligned}
$$

That is, the variation is performed solely with respect to the kinematic quantities. 
Let us define the strain-displacement matrix $\mathbf{B}$ which relates the reference strains with the displacements of control points for one isogeometric finite strip:

$$
\mathbf{e}=\mathbf{B q}=\mathbf{L H q}
$$

Required matrices are:

$$
\begin{aligned}
& \mathbf{L}=\left[\begin{array}{cccccccccccc}
x_{1,1} & 0 & x_{3,1} & 0 & 0 & 0 & 0 & 0 & 0 & 0 & 0 & 0 \\
0 & 0 & 0 & 0 & 1 & 0 & 0 & 0 & 0 & 0 & 0 & 0 \\
0 & 1 & 0 & x_{1,1} & 0 & x_{3,1} & 0 & 0 & 0 & 0 & 0 & 0 \\
-\Gamma_{11}^{1} x_{1,3} & 0 & -\Gamma_{11}^{1} x_{3,3} & 0 & 0 & 0 & x_{1,3} & x_{3,3} & 0 & 0 & 0 & 0 \\
0 & 0 & 0 & 0 & 0 & 0 & 0 & 0 & x_{1,3} & x_{3,3} & 0 & 0 \\
0 & 0 & 0 & 0 & 0 & 0 & 0 & 0 & 0 & 0 & 2 x_{1,3} & 2 x_{3,3}
\end{array}\right], \\
& \mathbf{H}=\left[\begin{array}{cccccc}
\mathbf{R}_{11} & \mathbf{R}_{12} & \cdots & \mathbf{R}_{I J} & \cdots & \mathbf{R}_{N M}
\end{array}\right], \\
& \mathbf{R}_{I J}^{\mathrm{T}}=\left[\begin{array}{lllll}
\mathbf{n}_{I J 1} & \mathbf{n}_{I J 2} & \mathbf{n}_{I J 11} & \mathbf{n}_{I J 22} & \mathbf{n}_{I J 12}
\end{array}\right],
\end{aligned}
$$

where the submatrices $\mathbf{n}_{I J}$ consist of the derivatives of basis functions for an arbitrary control point $I$ and series term $J$ :

$$
\begin{aligned}
\mathbf{n}_{I J 1} & =\left[\begin{array}{ccc}
R_{I, 1} f_{J}^{1} & 0 & 0 \\
0 & R_{I, 1} f_{J}^{2} & 0 \\
0 & 0 & R_{I, 1} f_{J}^{3}
\end{array}\right], \mathbf{n}_{I J 2}=\left[\begin{array}{ccc}
R_{I} f_{J, 2}^{1} & 0 & 0 \\
0 & R_{I} f_{J, 2}^{2} & 0 \\
0 & 0 & R_{I} f_{J, 2}^{3}
\end{array}\right], \\
\mathbf{n}_{I J 11} & =\left[\begin{array}{cc}
R_{I, 11} f_{J}^{1} & 0 \\
0 & 0 \\
0 & R_{I, 11} f_{J}^{3}
\end{array}\right], \mathbf{n}_{I J 22}=\left[\begin{array}{cc}
R_{I} f_{J, 22}^{1} & 0 \\
0 & 0 \\
0 & R_{I} f_{J, 22}^{3}
\end{array}\right], \mathbf{n}_{I J 12}=\left[\begin{array}{cc}
R_{I, 1} f_{J, 2}^{1} & 0 \\
0 & 0 \\
0 & R_{I, 1} f_{J, 2}^{3}
\end{array}\right] .
\end{aligned}
$$

Utilizing the Eqs. (45) and (48), the virtual work in expression (41) can be written as:

$$
\delta W=\int_{a} \mathbf{e}^{\mathrm{T}} \mathbf{D} \delta \mathbf{e} \mathrm{d} a+\rho h \int_{a} \ddot{\mathbf{i}}^{\mathrm{T}} \delta \mathbf{u} \mathrm{d} a=\mathbf{q}^{\mathrm{T}} \int_{a} \mathbf{B}^{\mathrm{T}} \mathbf{D} \mathbf{B} \mathrm{d} a \delta \mathbf{q}+\rho h \ddot{\mathbf{q}}^{\mathrm{T}} \int_{a} \mathbf{N}^{\mathrm{T}} \mathbf{N} \mathrm{d} a \delta \mathbf{q}=0,
$$

which is readily represented as:

$$
\begin{aligned}
& \mathbf{M} \ddot{\mathbf{q}}+\mathbf{K q}=0, \\
& \mathbf{K}=\int_{a} \mathbf{B}^{\mathrm{T}} \mathbf{D B} \mathrm{d} a, \quad \mathbf{M}=\rho h \int_{a} \mathbf{N}^{\mathrm{T}} \mathbf{N} \mathrm{d} a,
\end{aligned}
$$

where $\mathbf{K}$ and $\mathbf{M}$ are the stiffness and mass matrices of one isogeometric finite strip, respectively. The equation (52) is solved by assuming a synchronous and harmonic motion:

$$
\mathbf{q}=\tilde{\mathbf{q}} e^{i \omega t},
$$

which returns the following eigenvalue problem:

$$
\left(\mathbf{K}-\omega^{2} \mathbf{M}\right) \tilde{\mathbf{q}}=\mathbf{0},
$$

with $3 N M$ nontrivial solutions of eigenpairs of eigenfrequencies $\omega_{i}$ and corresponding eigenvectors $\tilde{\mathbf{q}}_{i}$.

\subsection{Series part of basis function}

As previously discussed, standard IGA employs the same basis functions for the description of geometry and kinematics. The present IGFSM, applied to the analysis of singly curved shells, uses series only for the kinematic field. This is analogous to the classic semi-analytical FSM where the eigenmodes of free transverse vibrations of the Bernoulli-Euler beam with problem-specific boundary conditions are usually 
employed for the description of kinematics, [20]. This approach enables modeling of all types of classic boundary conditions, [21]. In order to focus on the contributions of the NURBS-based interpolation, only the simply supported strips are implemented in this research. The other boundary conditions can be introduced in a straightforward manner.

For cylindrical shells, it is convenient to utilize two different series. The first approximates the two components of a displacement vector in a transverse plane of a shell. The second series approximates the longitudinal (along the $\eta$-axis) component of a displacement vector, Fig. 2, [21]. Let us assume that the longitudinal ends of a strip are supported with rigid diaphragms which restrain the displacement components in the transverse plane. For this case, a simple choice for the basis functions $f^{1}$ and $f^{3}$ is sine series:

$$
f_{J}^{1}=f_{J}^{3}=\sin (J \pi \eta / L), \quad J=1,2, \ldots M,
$$

which satisfies boundary conditions: $\hat{u}^{1}(\xi, 0)=\hat{u}^{1}(\xi, L)=\hat{u}^{3}(\xi, 0)=\hat{u}^{3}(\xi, L)=0$. Regarding the approximation of displacement component along the longitudinal direction, two different series are considered here:

$$
f_{J}^{2}=\left\{\begin{array}{l}
\sin (J \pi \eta / L) \\
\cos (J \pi \eta / L)
\end{array}, \quad J=1,2, \ldots M\right.
$$

The sine series fixes longitudinal displacements, $\hat{u}^{2}(\xi, 0)=\hat{u}^{2}(\xi, L)=0$, while the cosine series models free boundaries, $\hat{u}^{2}(\xi, 0)=-\hat{u}^{2}(\xi, L) \neq 0$. We will refer to these two types of supports as the rotationrigid diaphragms (RRD) and the rotation-flexible diaphragms (RFD), respectively.

\subsection{Coupled and uncoupled formulation}

One of the most prominent features of the original semi-analytical FSM comes from the orthogonality of employed beam eigenfunctions, [16]. If employed functions and their first and second derivatives are mutually orthogonal, the system equations uncouple with respect to the series terms, [18]. Let us simplify the notation with: $f_{m}^{1}=f_{m}^{3}=Z_{m}, f_{m}^{2}=Y_{m}$. In this way, the eight integrals with respect to the series terms which should be solved in the Eq. (52) are:

$$
\begin{aligned}
& I_{1 m n}=\int_{0}^{L} Z_{m} Z_{n} d \eta, I_{2 m n}=\int_{0}^{L} Z_{m, 22} Z_{n} d \eta, I_{3 m n}=\int_{0}^{L} Z_{m, 22} Z_{n, 22} d \eta, I_{4 m n}=\int_{0}^{L} Z_{m, 2} Z_{n, 2} d \eta, \\
& I_{5 m n}=\int_{0}^{L} Y_{m, 2} Z_{n} d \eta, I_{6 m n}=\int_{0}^{L} Y_{m} Z_{n, 2} d \eta, I_{7 m n}=\int_{0}^{L} Y_{m, 2} Y_{n, 2} d \eta, I_{8 m n}=\int_{0}^{L} Y_{m} Y_{n} d \eta .
\end{aligned}
$$

For the simple sine and cosine series, these integrals are readily solved analytically, even for more complex problems, [21]. A simple calculation shows that the system equations are uncoupled with respect to the series terms for the RFD, and coupled for the RRD supports, cf. Appendix. This uncoupling significantly reduces the required computational time because the calculation can be done with each series term independently.

\section{Numerical examples}

The purpose of the present numerical analysis is to verify and validate the developed IGFSM, and to examine its convergence properties. Since the exact solutions for the free vibrations of singly curved shells do not exist, the numerical reference solutions obtained with highly refined IGFSM meshes are utilized in each experiment. A designation for an IGFSM mesh is $m \mathrm{~h}-n \mathrm{p}-i \mathrm{k}-j \mathrm{t}$, where $m, n, i$, and $j$ are the number of strips (knot spans), the order of NURBS, the interstrip continuity, and the number of 
series terms, respectively. In general, the IGFSM model has $3 N M$ DOFs. For the models with RFD supports, the number of series terms equals the highest number of half-waves along the longitudinal direction which appears in the spectrum being observed. On the contrary, more series terms are required for the RRD models due to the coupling between the eigenmodes.

The quadrature rules are of special importance in the IGA due to the increased smoothness of basis functions across element boundaries, [42]. In our code, the integration of NURBS functions is performed on the element level by employing standard Gauss quadrature with $p+1$ integration points. It should be noted that more efficient techniques for the assembly of system matrices in IGA exist, [43]. Nevertheless, the standard approach is well-suited for the purpose of validating the new method and it is applied here. Regarding the longitudinal direction, the integration of trigonometric functions is done analytically, cf. Appendix.

The first example deals with a circular cylinder which has been widely researched and several numerical and experimental results are available. The other three studies are conducted on the shells with antisymmetrical and symmetrical cross-sections. These shells are discussed here in order to emphasize the versatility of the newly developed IGFSM. The validation tests are done by the comparison with the results found with dense uniform meshes of STRI3 shell elements. This element is the only one in the Abaqus library which imposes KL constraints analytically, [44]. For each example, the results of two different FE meshes are given for illustration and reproduction purposes only. The efficiency comparison of the derived FSIGA strip with a general shell element, such as the STRI3, is beyond the scope of this paper.

Additional comparison is made with the standard flat shell finite strips LO2 and HO3, [18], [21]. Both strips have four DOFs per nodal line: three displacement components and infinitesimal rotation around the longitudinal axis. The LO2 strip employs Hermite cubic polynomials for transverse displacements, and linear polynomials for in-plane displacements. The HO3 strip has one internal auxiliary nodal line and the interpolating polynomials are of the fifth and second order. Both strips have $C^{l}$ interstrip continuity.

\subsection{An open circular cylinder}

A well-investigated example of an open circular cylinder with RFD supports is studied first, Fig. 3. The reference IGFSM frequencies for the first eight modes are calculated with $80 \mathrm{~h}-4 \mathrm{p}-1 \mathrm{k}-3 \mathrm{t}$ mesh, and given in Table 1. A close agreement between the present results and those reported in literature is evident. Also, the frequencies are close to the experimental values obtained in [45]. It should be noted that some of the cited references did not report the fifth mode. Regarding the mode shapes, they are virtually indistinguishable from those in Abaqus and literature, and only the IGFSM results are visualized, Fig. 4. The highest number of the transverse sine half-waves along the longitudinal direction is 3 , for the part of the spectrum observed in this study. This suggest that only 3 series terms are needed to obtain accurate IGFSM results. The efficiency of the developed method is clearly pronounced since the highly refined solutions are obtained by employing a comparatively small number of DOFs, Table 1.

The convergence properties of the IGFSM are examined next. The results of $h$-refinement tests are given in Fig. 5, Fig. 6, and Fig. 7. The orders of convergence are estimated since they generally oscillate with respect to the $h$-refinement. This phenomenon is more pronounced for more complex initial geometries, [30]. The results imply that an increase in the NURBS order and interstrip continuity significantly increases the order of convergence. Moreover, a close inspection of the results in Fig. 6 reveals that models with high interstrip continuity have improved accuracy per DOF. The convergences of LO2 and $\mathrm{HO} 3$ strips are displayed in Fig. 7. It is evident that these flat shell strips have inferior orders of convergence in comparison with the curved NURBS strips of similar order. 
The effect of $p$-refinement is explicitly demonstrated in Fig. 8 where the relative error of frequencies is scrutinized versus the order of NURBS functions. As predicted, relative error decreases with the increase in the order of NURBS, Fig. 8a. Still, the relative errors normed with the number of DOFs show some unexpected results, Fig. 8b. Namely, the accuracy per DOF for the first and sixth eigenfrequencies drops as the order changes from $p=3$ to $p=4$. A similar behavior is already detected for plane beams in [30]. It is argued that there is an optimal order of NURBS for each mode, in the terms of accuracy per DOF.

Finally, the $k$-refinement is studied directly and the results are visualized in Fig. 9. The relative errors oscillate with respect to the interstrip continuity, Fig. 9a. On the contrary, the relative error normed with the number of DOFs decreases monotonically, Fig. 9b. These results clearly indicate that the models with higher interstrip continuities are more efficient. Furthermore, it is found that the differences between normed relative errors increase with the increase in continuity, Fig. 9b. This is primarily due to the reduced number of DOFs for $k$-refined meshes. Additionally, high interstrip continuity is more beneficial for smoother structural responses, such as the $1^{\text {st }}$ and $2^{\text {nd }}$ modes in Fig. 4. Finally, it is worth noting how the frequency of the fifth mode is practically invariant to the change of continuity, Fig. 9a.

It should be pointed out that a general insight into the effects of $p$-and $k$-refinements can be made indirectly, by observing the $h$-refinement studies with the different orders of NURBS and interstrip continuities, such as in Fig. 5 and Fig. 6. In addition, the impact of refinements on convergence is more involved for non-circular initial geometries, [30]. Therefore, the explicit presentation of $p$ - and $k$ refinement techniques is omitted in the next two examples.

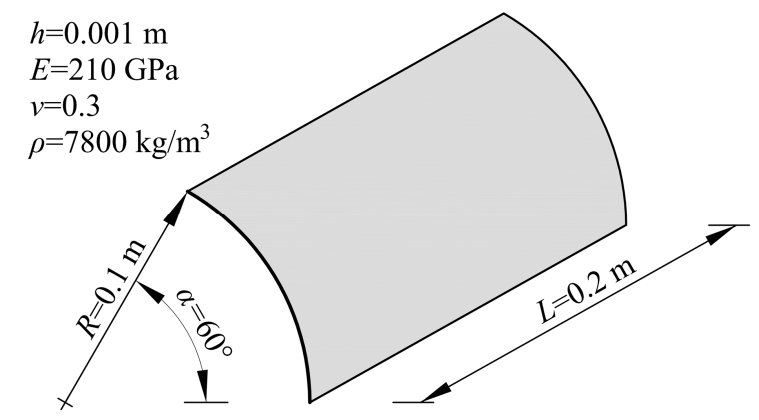

Fig. 3. The open circular cylinder.

Table 1. The open circular cylinder. Comparison of the lowest eight eigenfrequencies [Hz].

\begin{tabular}{cccccccc} 
Mode & Ref. [5] & Ref. [12] & $\begin{array}{c}\text { Ref. } \\
{[11]}\end{array}$ & $\begin{array}{c}\text { Ref. [45] } \\
\text { Experimental }\end{array}$ & $\begin{array}{c}\text { Abaqus STRI3 } \\
\text { elements } \\
\left(\mathrm{n}_{\text {DOF }}=5412\right)\end{array}$ & $\begin{array}{c}167200 \\
\text { elements } \\
\left(\mathrm{n}_{\text {DOF }}=505260\right)\end{array}$ & $\begin{array}{c}\text { P0h-4p-1k-3t } \\
\left(\mathrm{n}_{\text {DOF }}=2178\right)\end{array}$ \\
\hline 1 & 285.162 & 286.012 & 286 & 300 & 295.03 & 286.18 & 285.99 \\
2 & 475.131 & 476.367 & 476 & 470 & 478.97 & 476.31 & 476.30 \\
3 & 817.6 & 819.124 & 819 & 850 & 836.00 & 819.34 & 818.99 \\
4 & 857.365 & 859.198 & 859 & 870 & 885.37 & 859.90 & 859.38 \\
5 & & 952.465 & & & 961.43 & 952.30 & 952.12 \\
6 & 1339.58 & 1341.65 & 1341 & 1330 & 1382.4 & 1342.4 & 1341.6 \\
7 & 1437.56 & 1440.09 & 1440 & 1460 & 1469.7 & 1441.0 & 1440.2 \\
8 & 1486.98 & & 1486 & 1490 & 1476.4 & 1485.2 & 1486.0 \\
\hline
\end{tabular}



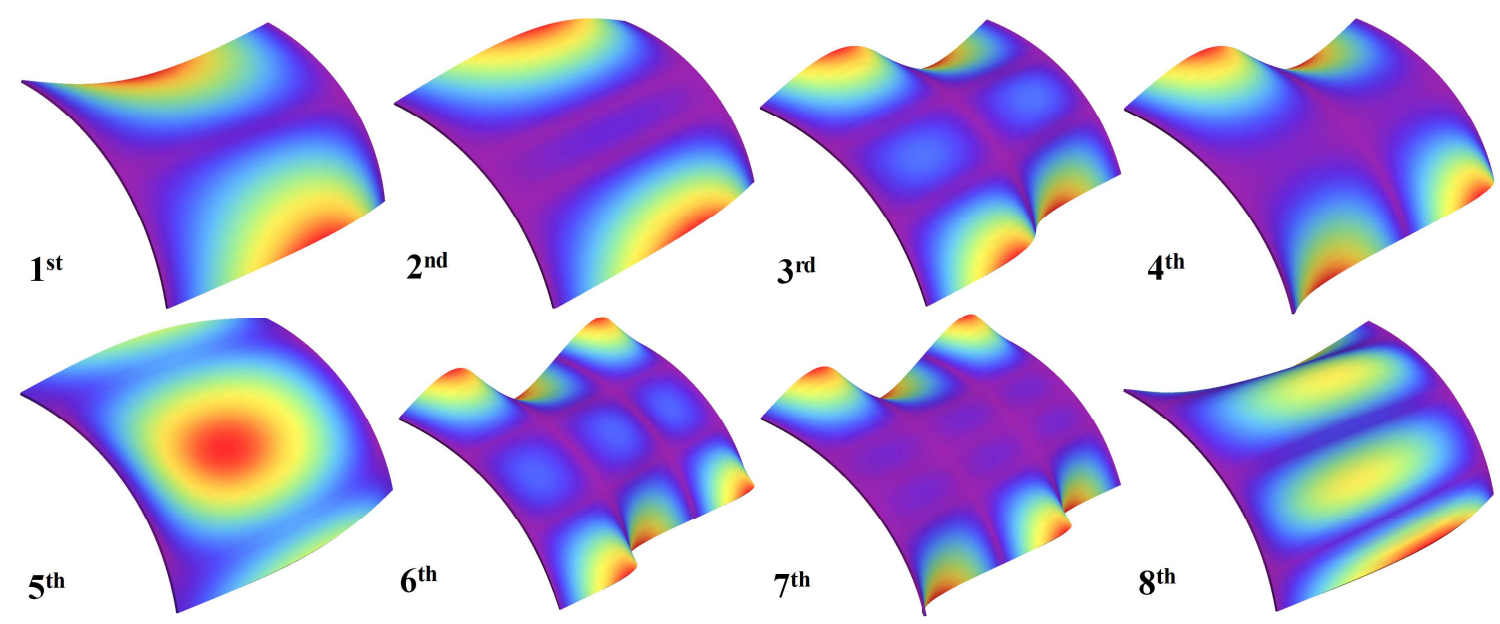

Fig. 4. The open circular cylinder. Mode shapes of the lowest eight modes.
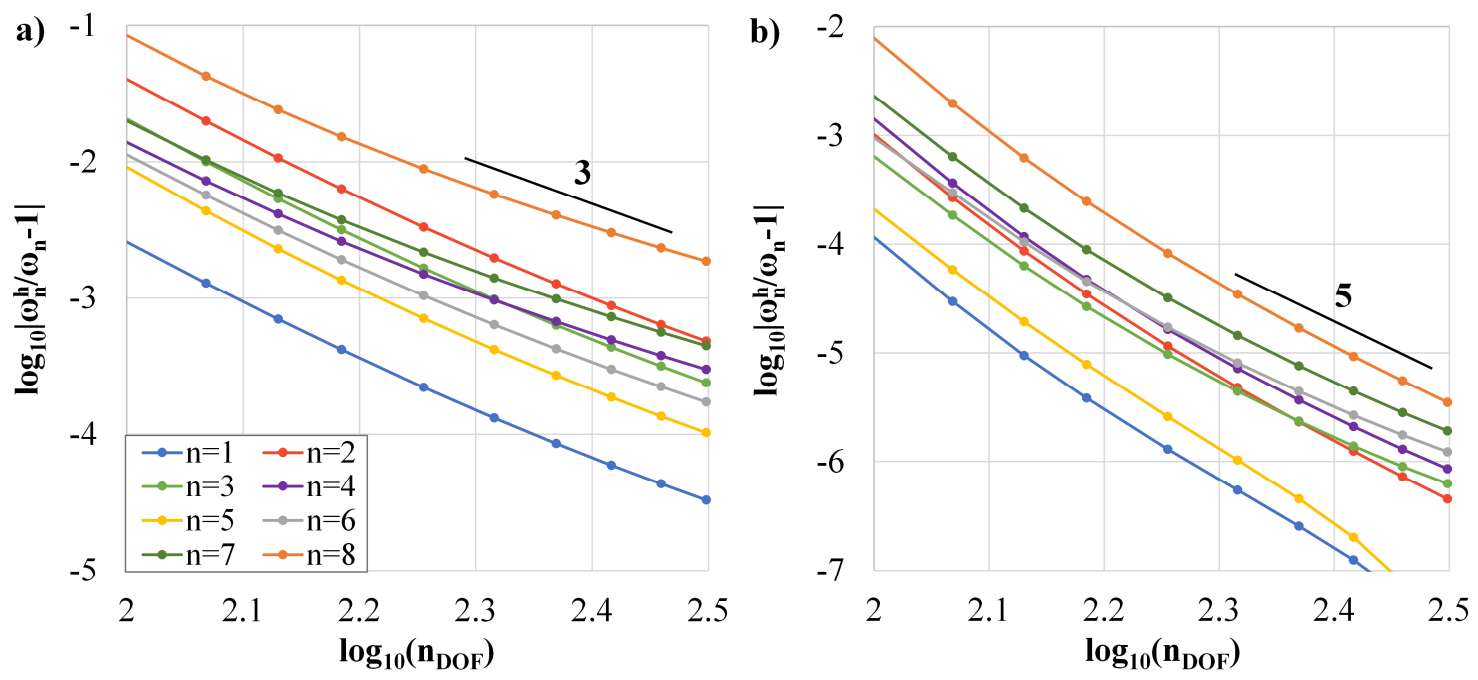

Fig. 5. The open circular cylinder. Convergence of the relative error for the lowest eight eigenfrequencies using the $h$-refinement: a) $m \mathrm{~h}-2 \mathrm{p}-1 \mathrm{k}-3 \mathrm{t}$; b) $m \mathrm{~h}-3 \mathrm{p}-2 \mathrm{k}-3 \mathrm{t}$.
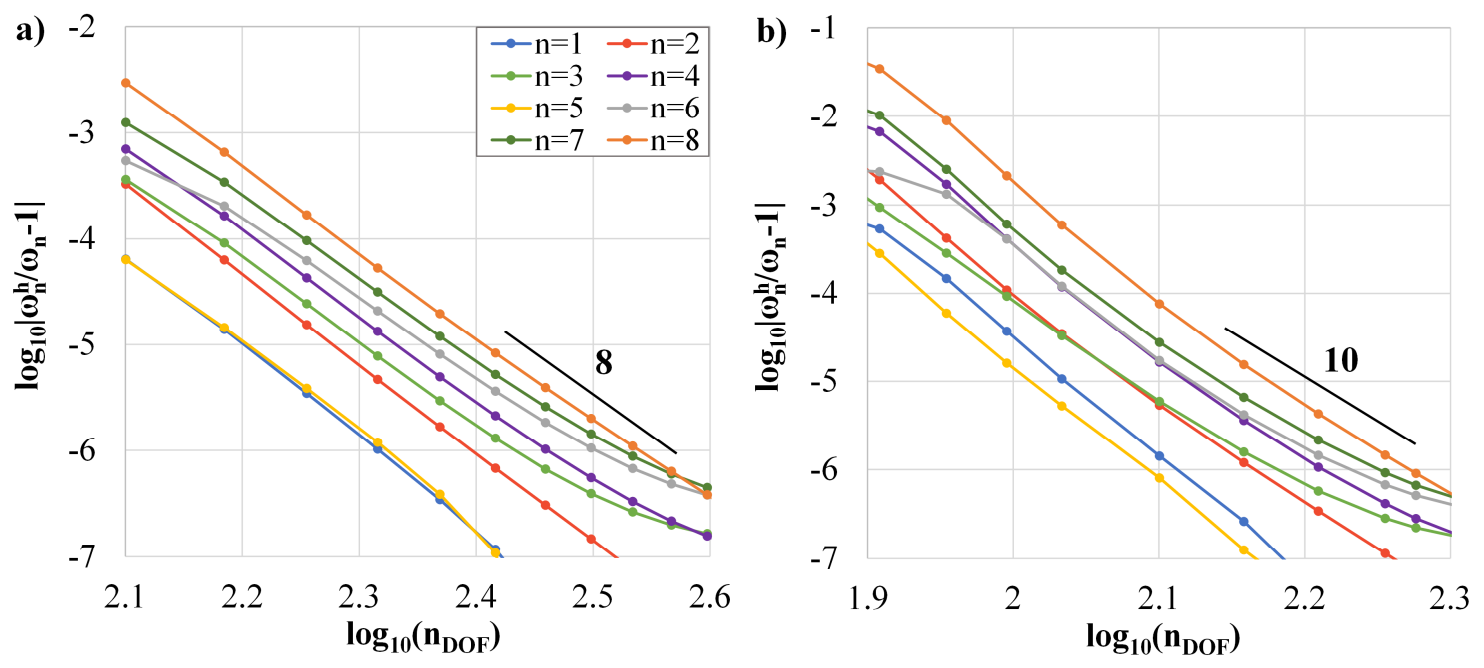

Fig. 6. The open circular cylinder. Convergence of the relative error for the lowest eight eigenfrequencies using the $h$-refinement: a) $m \mathrm{~h}-4 \mathrm{p}-1 \mathrm{k}-3 \mathrm{t}$; b) $m \mathrm{~h}-4 \mathrm{p}-3 \mathrm{k}-3 \mathrm{t}$. 

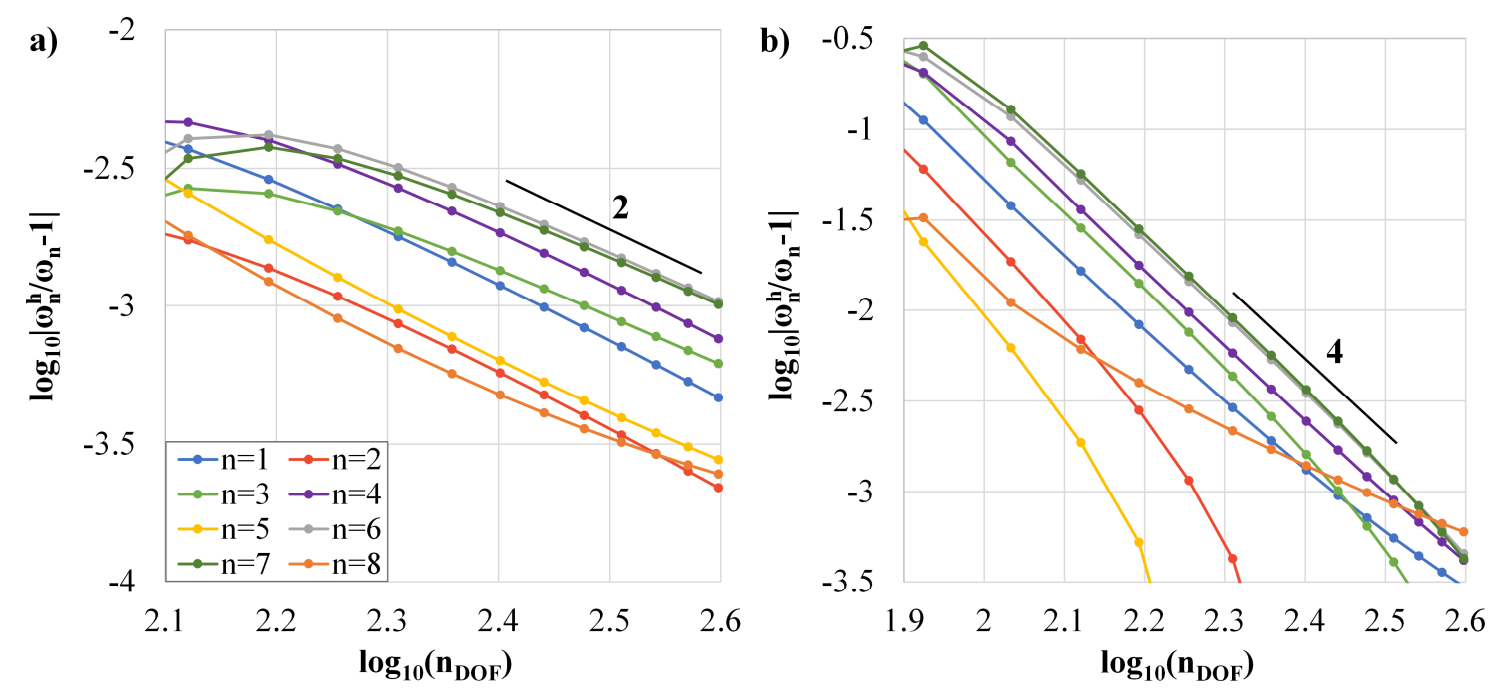

Fig. 7. The open circular cylinder. Convergence of the relative error for the lowest eight eigenfrequencies using the $h$-refinement and three seres terms: a) LO2 strip; b) HO3 strip.
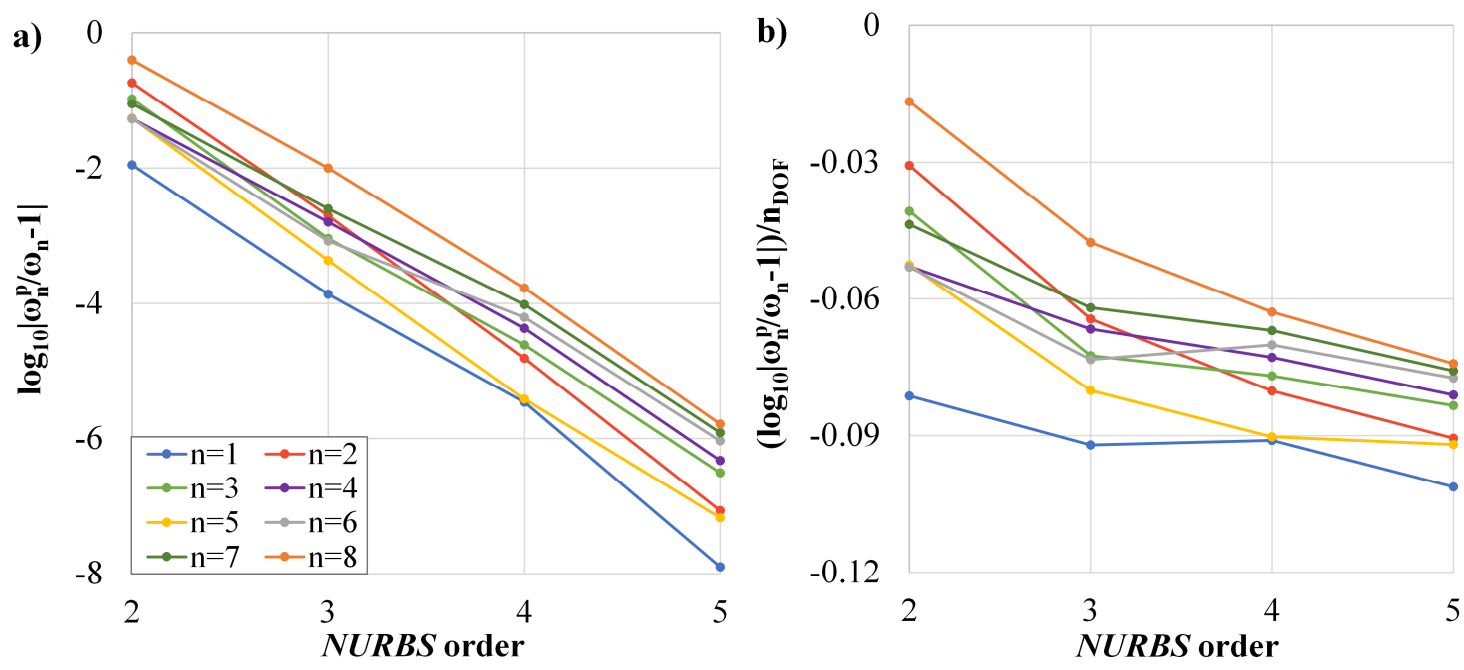

Fig. 8. The open circular cylinder. Convergence of the relative error for the lowest eight eigenfrequencies using the $p$-refinement with the meshes $6 \mathrm{~h}-n \mathrm{p}-1 \mathrm{k}-3 \mathrm{t}$ : a) the relative error; b) the relative error divided with the number of DOFs.
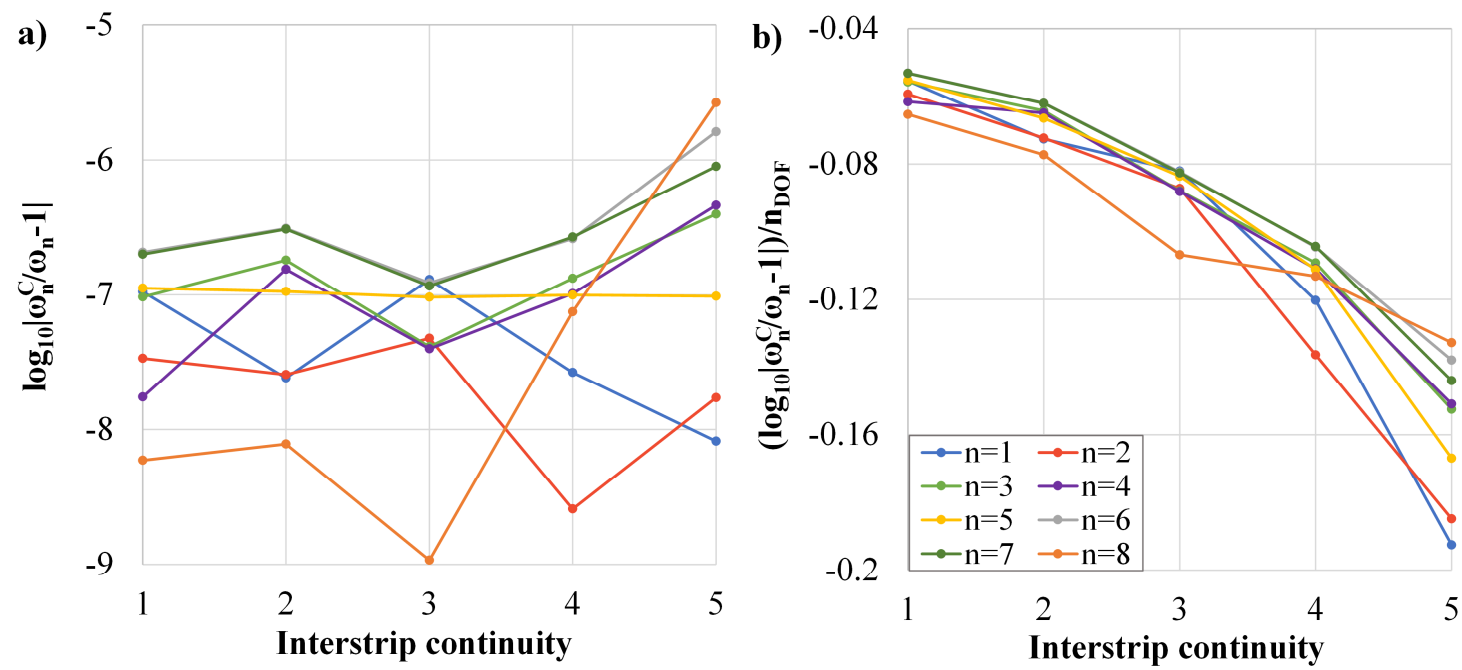
Fig. 9. The open circular cylinder. Convergence of the relative error for the lowest eight eigenfrequencies using the $k$-refinement with the meshes $8 \mathrm{~h}-6 \mathrm{p}-n \mathrm{k}-3 \mathrm{t}$ : a) the relative error; b) the relative error divided with the number of DOFs.

\subsection{A cubic cylinder with antisymmetric cross section}

A cylinder with the circumferential profile described by the cubic B-spline is displayed in Fig. 10a. A B-spline is the special case of NURBS where all weights are set to 1, [39]. The parametric equations of this curve are:

$$
x(\xi)=1.2 \xi-2.4 \xi^{2}+1.6 \xi^{3}, \quad z(\xi)=1.5 \xi^{2}-\xi^{3} .
$$

Distribution of the modulus of curvature is shown in Fig. 10b. Since the maximum curvature is near 8 , the maximum curviness for this shell is $K h \approx 0.08$.

The reference results are calculated with $80 \mathrm{~h}-4 \mathrm{p}-1 \mathrm{k}-3 \mathrm{t}$ mesh for the RFD, and with $80 \mathrm{~h}-4 \mathrm{p}-1 \mathrm{k}-$ $40 \mathrm{t}$ mesh for the RRD supports. The eigenfrequencies are compared in Table 2 while the mode shapes are displayed in Fig. 11 and Fig. 12. The results obtained with the IGFSM show close agreement with those from the Abaqus simulations. The benefit of uncoupling is particularly emphasized because the reference RFD model has less DOFs than the RRD one by the order of magnitude.

The mode shapes are the same as those in Abaqus and their detail examination is a challenging task. Some types of global, local, and distortional mode shapes are observed. However, due to the relative flexibility of the circumferential profile, these modes are not as clear as for the classic coldformed thin-walled structures with high values of local curvatures. A more detailed discussion requires the application of some rigorous classification technique, such as the one implemented in the constrained FSM, [46].

The results of $h$-refinement are given in Fig. 13, Fig. 14, Fig. 15, Fig. 16, and Fig. 17. As expected, improved orders of convergence are found for the models with higher orders of B-spline and/or the increased interstrip continuity. The models with RFD boundary conditions have excellent accuracy per DOF due to the decoupling of modes. In comparison with the previous example, the orders of convergence for the RFD model and quartic NURBS are lower in this example. This is attributed to the influence of more complex reference geometry. The effect of $h$-refinement for $\mathrm{LO} 2$ and HO3 strips and RRD supports is presented in Fig. 17. The LO2 strip gives a similar order of convergence as in the previous example, while the order of $\mathrm{HO} 3$ strip is significantly deteriorated, due to the more complex initial geometry.

For the cylinder with RRD supports, a $t$-refinement results are displayed in Fig. 18 for cubics and quartics with the highest continuities. Similar orders of convergence are estimated for both models, near 2.5. It is interesting to note the influence of odd and even series terms. It could be expected that symmetric eigenfunctions do not affect antisymmetric modes, and vice versa. Although this is true, but the modes still depend on both transversal and longitudinal displacement components. In the present formulation, the longitudinal membrane displacements are generally antisymmetric for symmetric flexural modes, and symmetric for antisymmetric flexural modes, Eqs. (55) and (56). Due to this fact, both odd and even series terms influence both types of modes, Fig. 18. Finally, influence of $t$-refinement for $\mathrm{LO} 2$ and HO3 strips is shown in Fig. 19. The results are practically the same as those in Fig. 18 due to the dense mesh of strips in all these tests. 

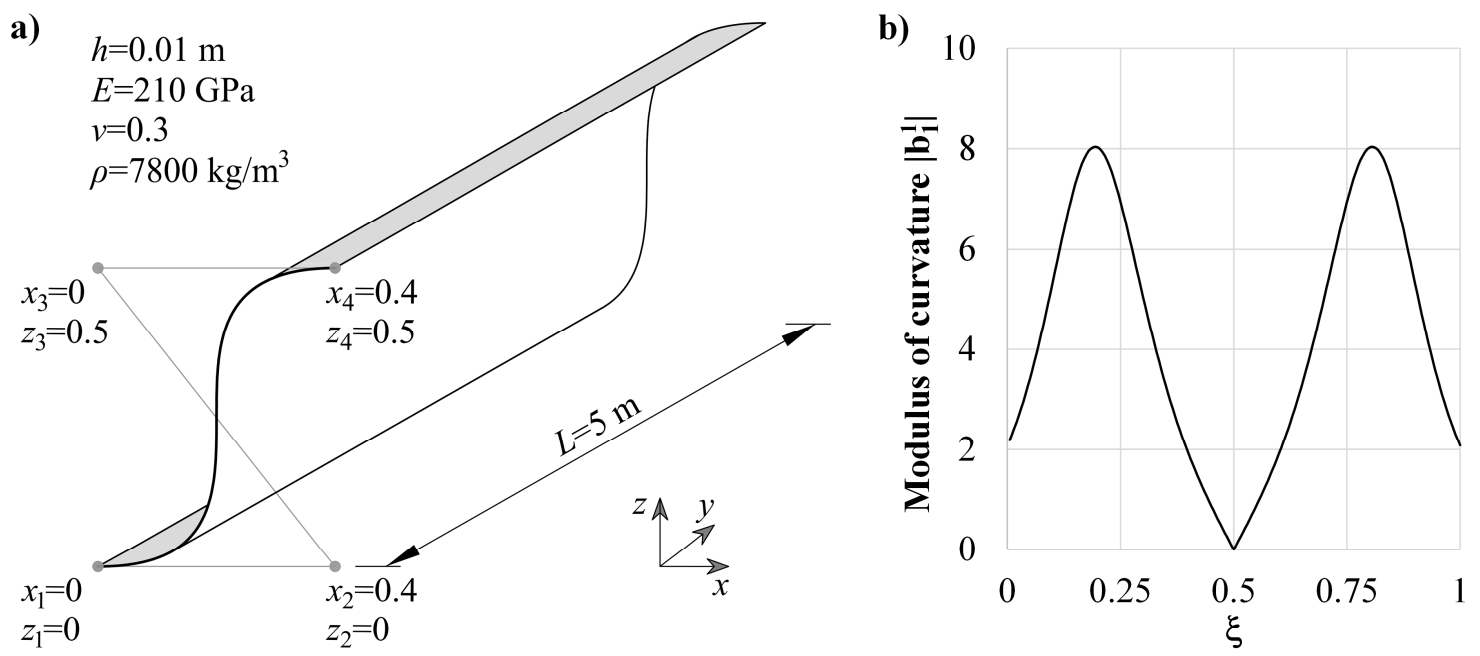

Fig. 10. The cubic cylinder. a) Geometry and material properties. b) Distribution of the modulus of curvature along $\xi$ coordinate.

Table 2. The cubic cylinder. Comparison of the lowest eight eigenfrequencies [Hz].

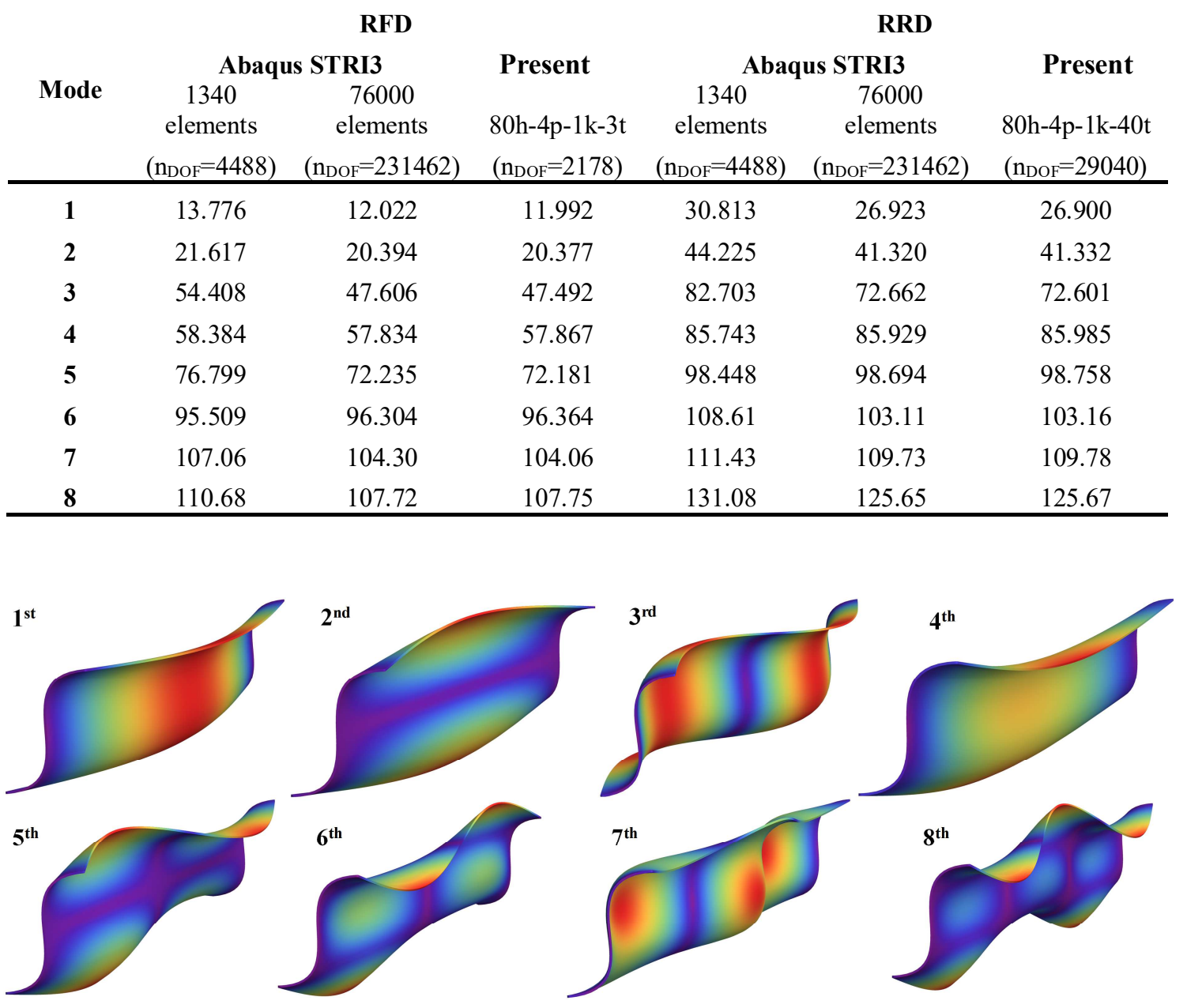

Fig. 11. The cubic cylinder with RFD supports. Mode shapes of the lowest eight modes. 


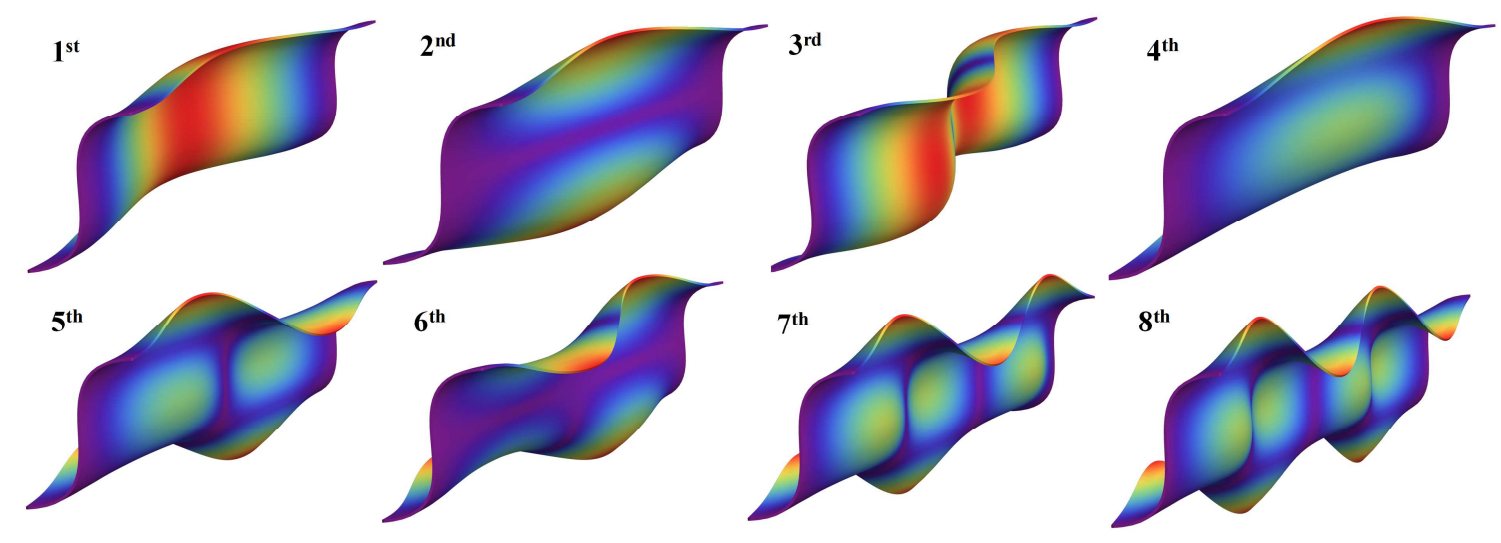

Fig. 12. The cubic cylinder with RRD supports. Mode shapes of the lowest eight modes.
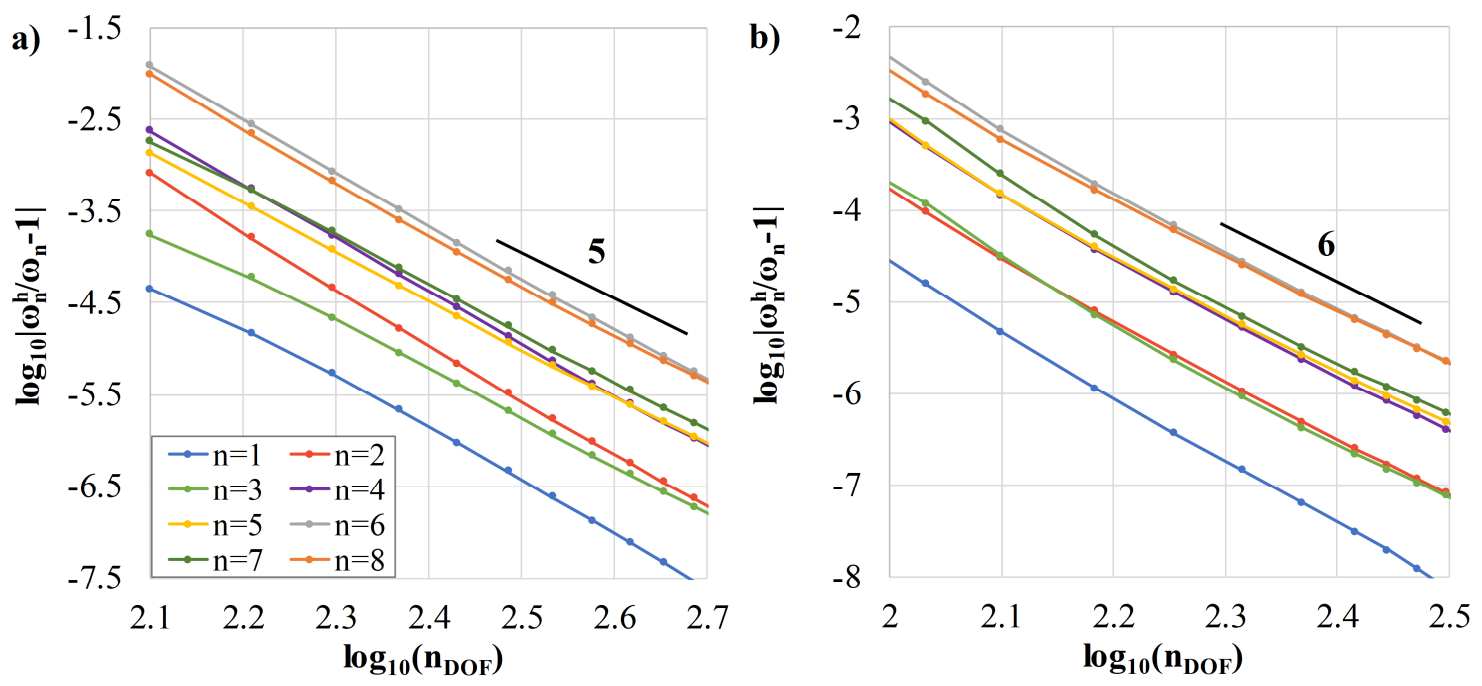

Fig. 13. The cubic cylinder with RFD supports. Convergence of the relative error for the lowest eight eigenfrequencies using the $h$-refinement: a) $m \mathrm{~h}-3 \mathrm{p}-1 \mathrm{k}-3 \mathrm{t}$; b) $m \mathrm{~h}-3 \mathrm{p}-2 \mathrm{k}-3 \mathrm{t}$.
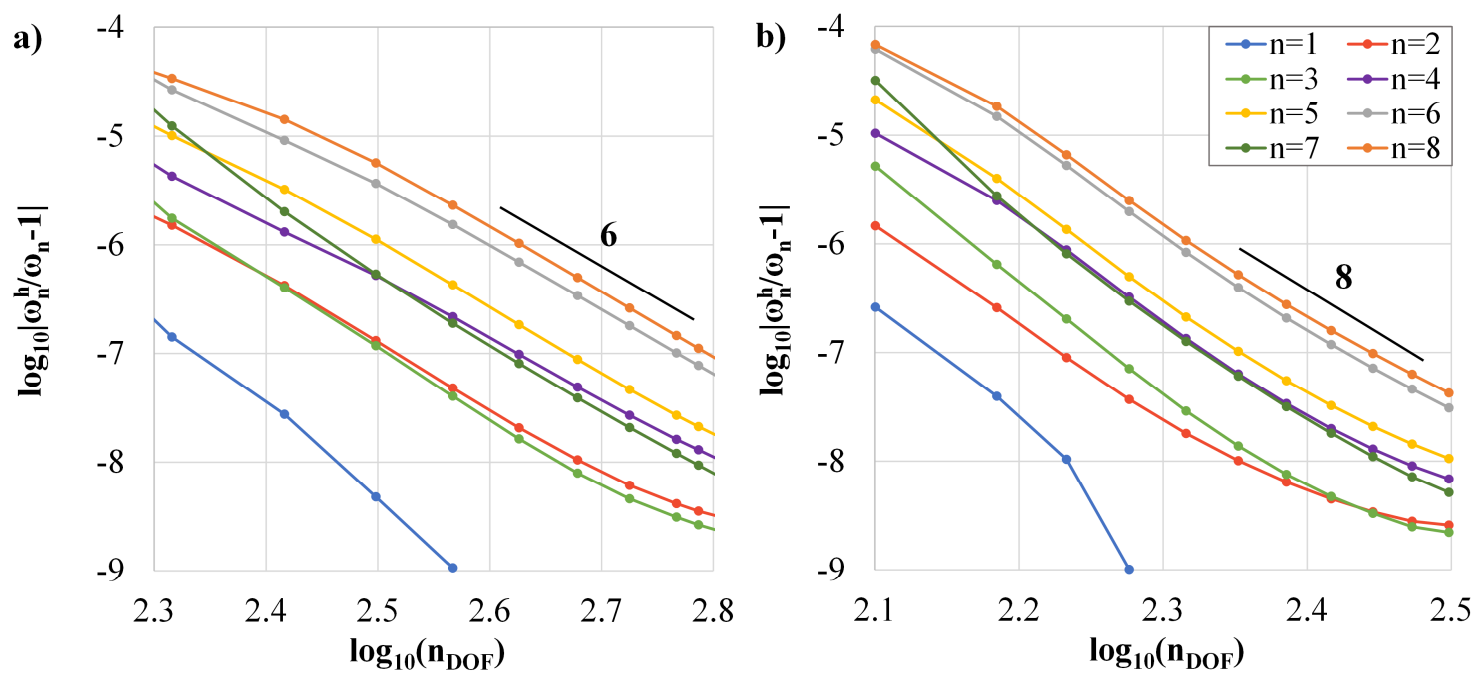

Fig. 14. The cubic cylinder with RFD supports. Convergence of the relative error for the lowest eight eigenfrequencies using the $h$-refinement: a) $m \mathrm{~h}-4 \mathrm{p}-1 \mathrm{k}-3 \mathrm{t}$; b) $m \mathrm{~h}-4 \mathrm{p}-3 \mathrm{k}-3 \mathrm{t}$. 

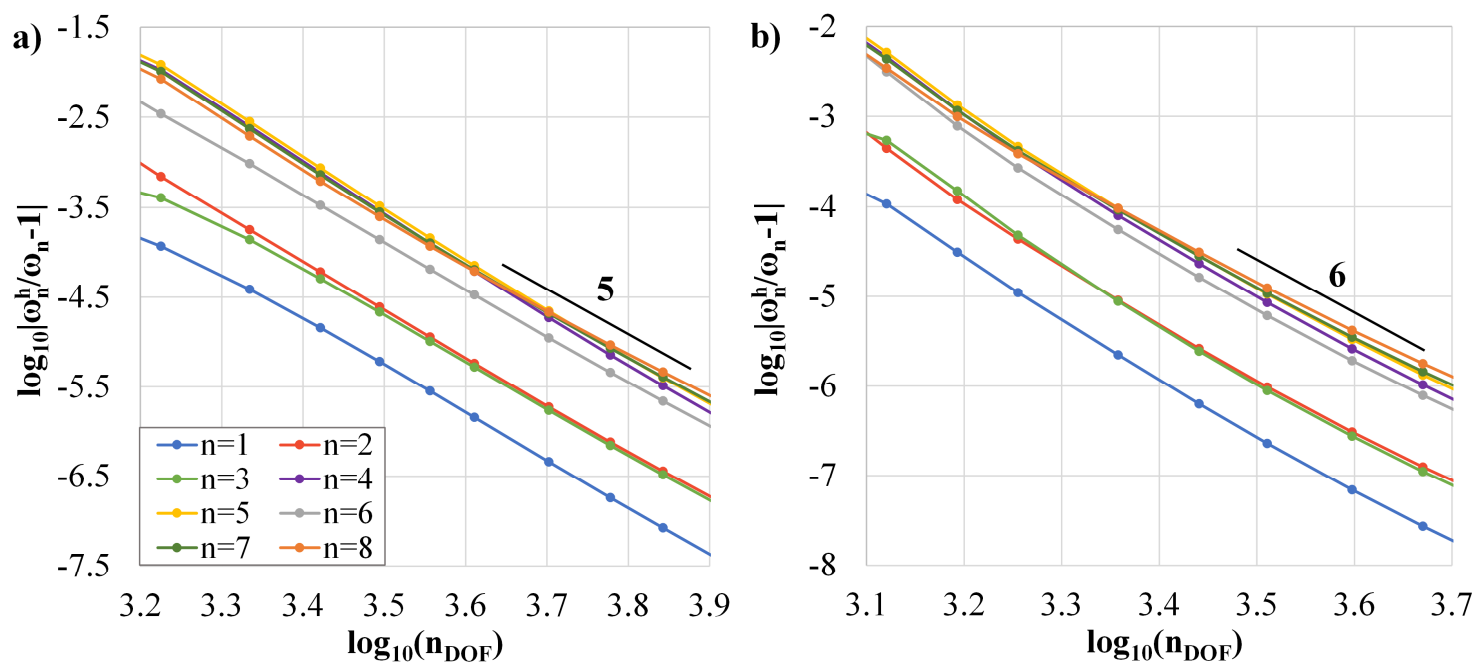

Fig. 15. The cubic cylinder with RRD supports. Convergence of the relative error for the lowest eight eigenfrequencies using the $h$-refinement: a) $m \mathrm{~h}-3 \mathrm{p}-1 \mathrm{k}-40 \mathrm{t}$; b) $m \mathrm{~h}-3 \mathrm{p}-2 \mathrm{k}-40 \mathrm{t}$.
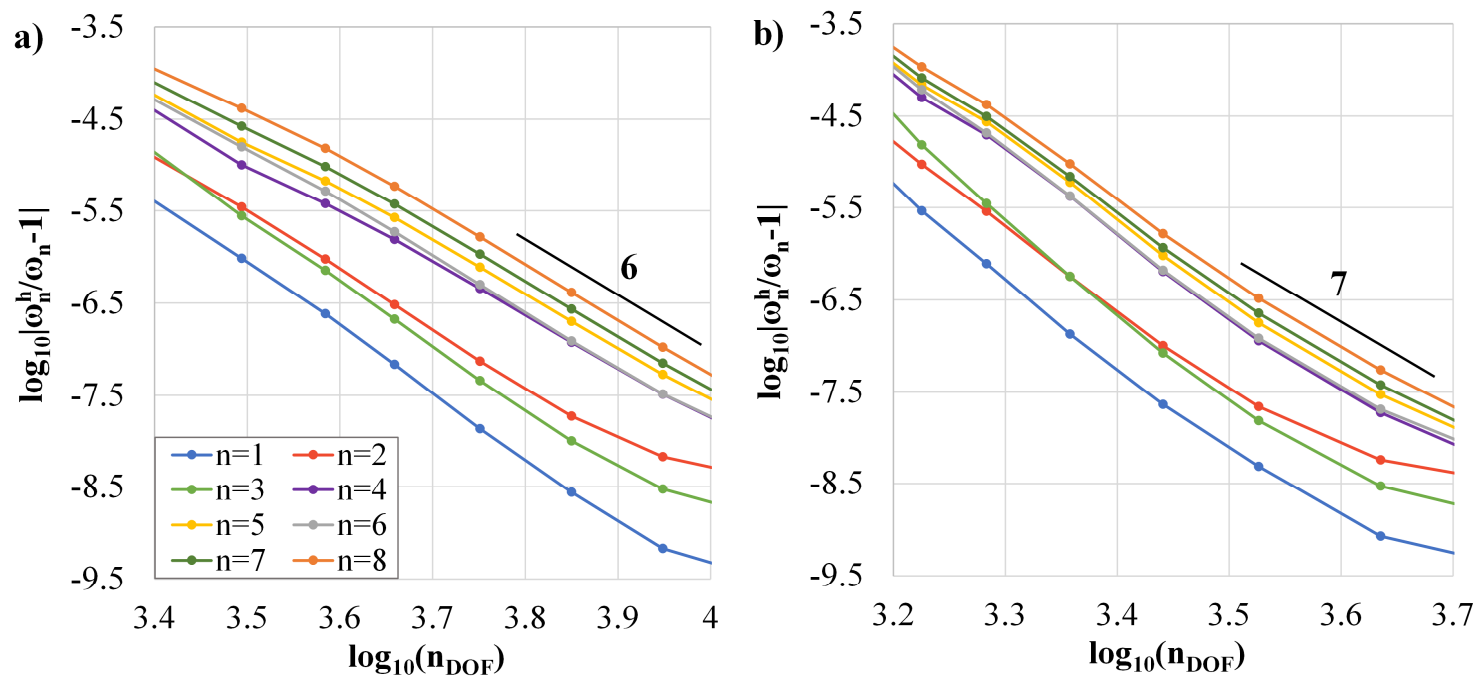

Fig. 16. The cubic cylinder with RRD supports. Convergence of the relative error for the lowest eight eigenfrequencies using the $h$-refinement: a) $m \mathrm{~h}-4 \mathrm{p}-1 \mathrm{k}-40 \mathrm{t}$; b) $m \mathrm{~h}-4 \mathrm{p}-3 \mathrm{k}-40 \mathrm{t}$.
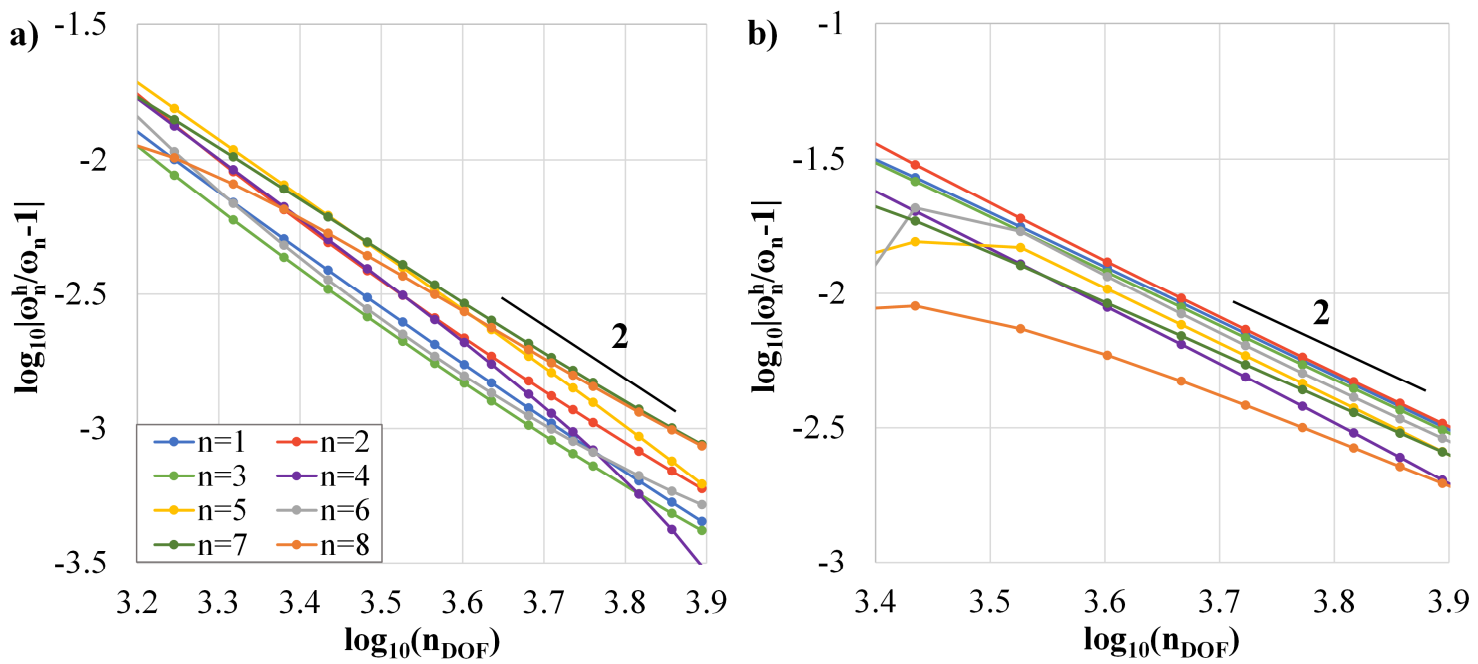

Fig. 17. The cubic cylinder with RRD supports. Convergence of the relative error for the lowest eight eigenfrequencies using the $h$-refinement and 40 series terms: a) LO2 strip; b) HO3 strip. 

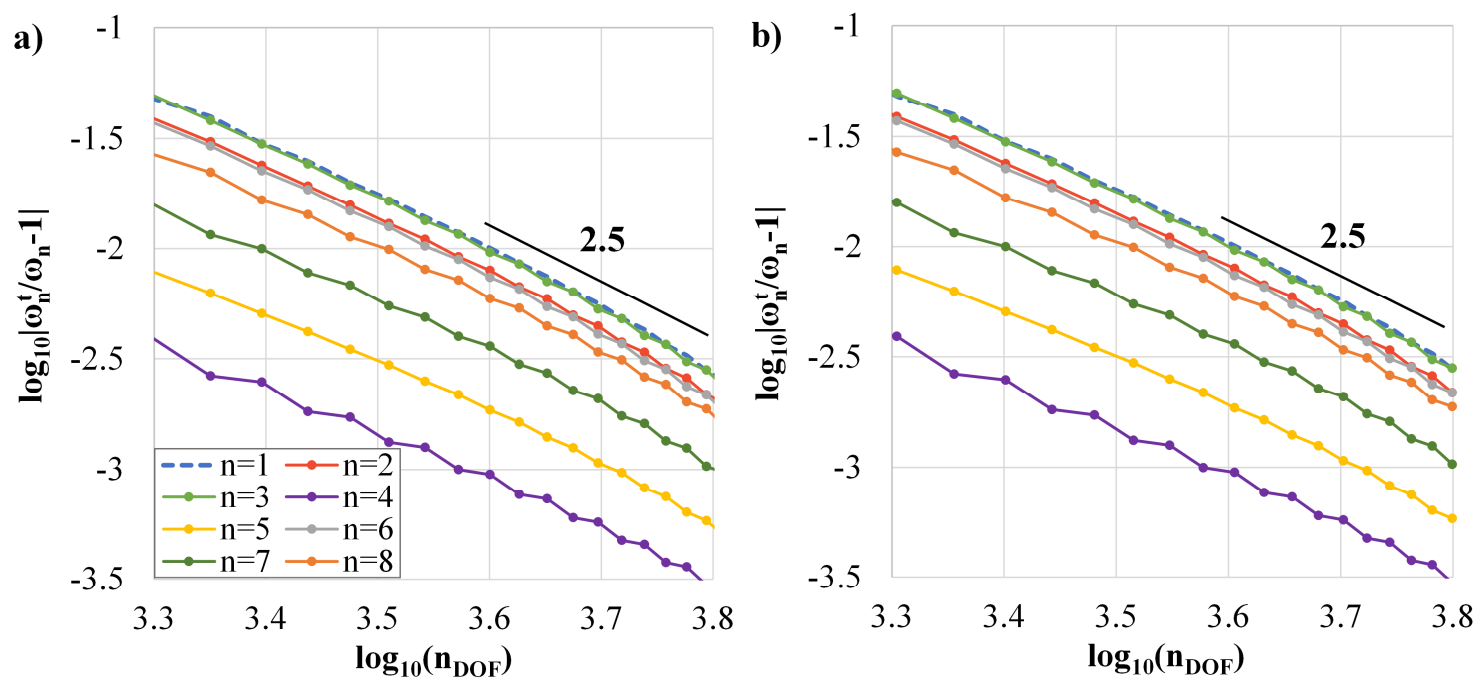

Fig. 18. The cubic cylinder with RRD supports. Convergence of the relative error for the lowest eight eigenfrequencies using the $t$-refinement: a) $80 \mathrm{~h}-3 \mathrm{p}-2 \mathrm{k}-j \mathrm{t}$; b) $80 \mathrm{~h}-4 \mathrm{p}-3 \mathrm{k}-j \mathrm{t}$.
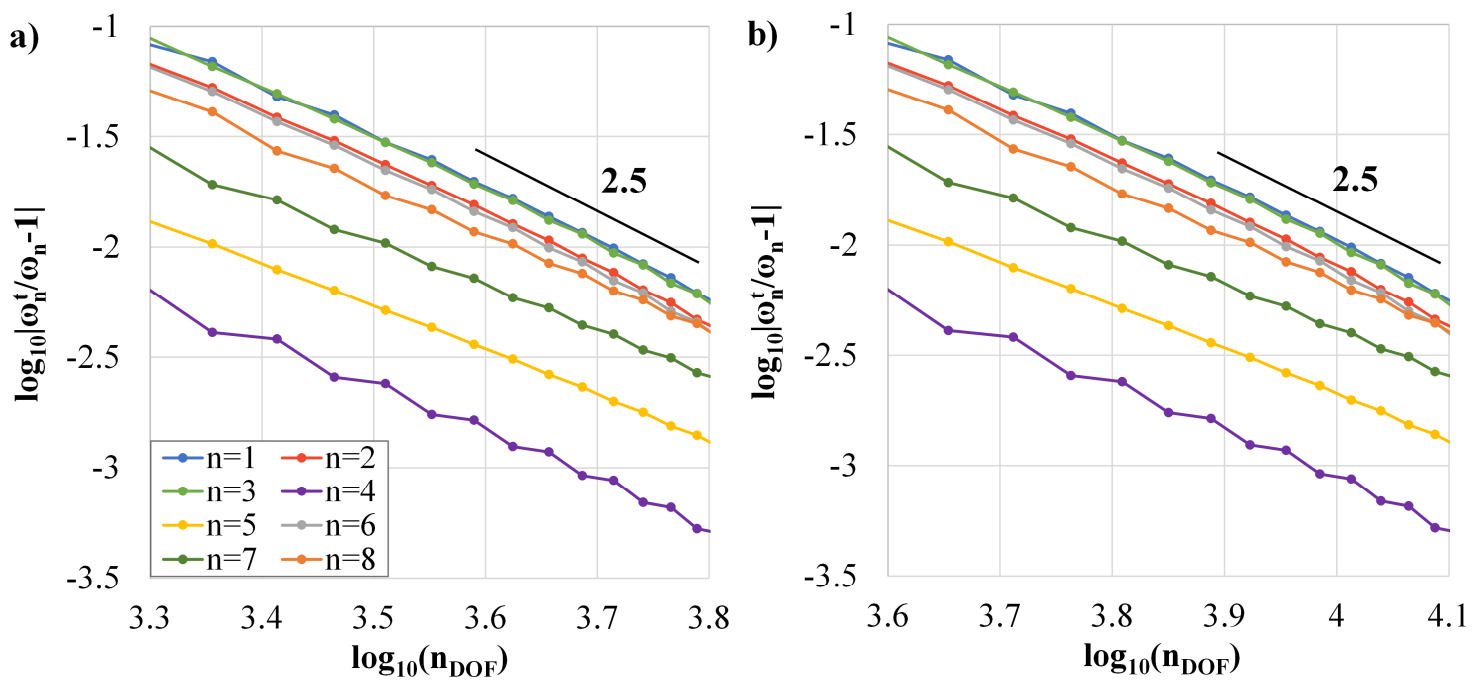

Fig. 19. The cubic cylinder with RRD supports. Convergence of the relative error for the lowest eight eigenfrequencies using the $t$-refinement and 80 strips: a) LO2 strip; b) HO3 strip.

\subsection{A quartic cylinder with symmetric cross section}

A shell with symmetric circumferential profile, described with the quartic NURBS, is considered in this numerical experiment, Fig. 20a. Its parametric equations are:

$$
x(\xi)=\frac{0.08-0.32 \xi+1.2 \xi^{2}-1.76 \xi^{3}+0.88 \xi^{4}}{0.2+0.8 \xi-1.8 \xi^{2}+2 \xi^{3}-\xi^{4}}, \quad z(\xi)=\frac{-0.45 \xi^{2}+0.1 \xi^{3}+0.25 \xi^{4}}{-0.2-0.8 \xi+1.8 \xi^{2}-2 \xi^{3}+\xi^{4}} .
$$

The reference meshes are the same as in the previous example, except the 4 series terms are used for the RFD model, instead of 3. Distribution of the modulus of curvature is displayed in Fig. 20b. Since the maximum value of curvature is close to 16 , the maximum curviness $K h$ is practically the same as for the cubic shell.

The lowest eight eigenfrequencies are scrutinized with respect to the results obtained with STRI3 elements, Table 3. Again, an excellent accuracy per DOF of the present method is observed. This feature is especially pronounced for the shell with RFD supports where only four series terms are required to obtain accurate results. It is remarked that almost all the eigenfrequencies of this cylinder 
are lower than those of the cubic cylinder. The lowest two modes for the RFD and the first mode for the RRD supports are the exceptions. The mode shapes are presented in Fig. 21 and Fig. 22 and all the comments from the previous example hold.

The convergences of eigenfrequencies with respect to the $h$-refinement are presented in Fig. 23 and Fig. 24. These graphs clearly show how the oscillation of the orders of convergence gets more pronounced as the initial geometry becomes more complicated. Namely, the distributions of curvatures in Fig. $10 \mathrm{~b}$ and Fig. $20 \mathrm{~b}$ prove that the initial geometry of the quartic shell changes more swiftly, compared to the cubic one. As in both previous examples, the effects of increased NURBS order and interstrip continuity are clearly indicated. However, the orders of convergence are lower than those for the cubic shell. Again, this is due to the more complex initial geometry of the quartic cylinder.

The $t$-refinement is considered at the end, Fig. 25. The orders of convergences are similar to those estimated in the previous example, close to 2.5. This is due to the same, straight, initial geometry along the longitudinal direction in both examples. The phenomenon of the oscillatory order of convergence is visible again. As explained, this effect is caused by the coupling of odd and even series terms. Close inspection of Fig. 18, Fig. 19, and Fig. 25 reveals that the $t$-refinement is invariant with respect to the other mesh refinement techniques. Finally, similar values of the relative errors in Fig. 25a and Fig. 25b prove the benefit of high interstrip continuity for utilized quartic NURBS.
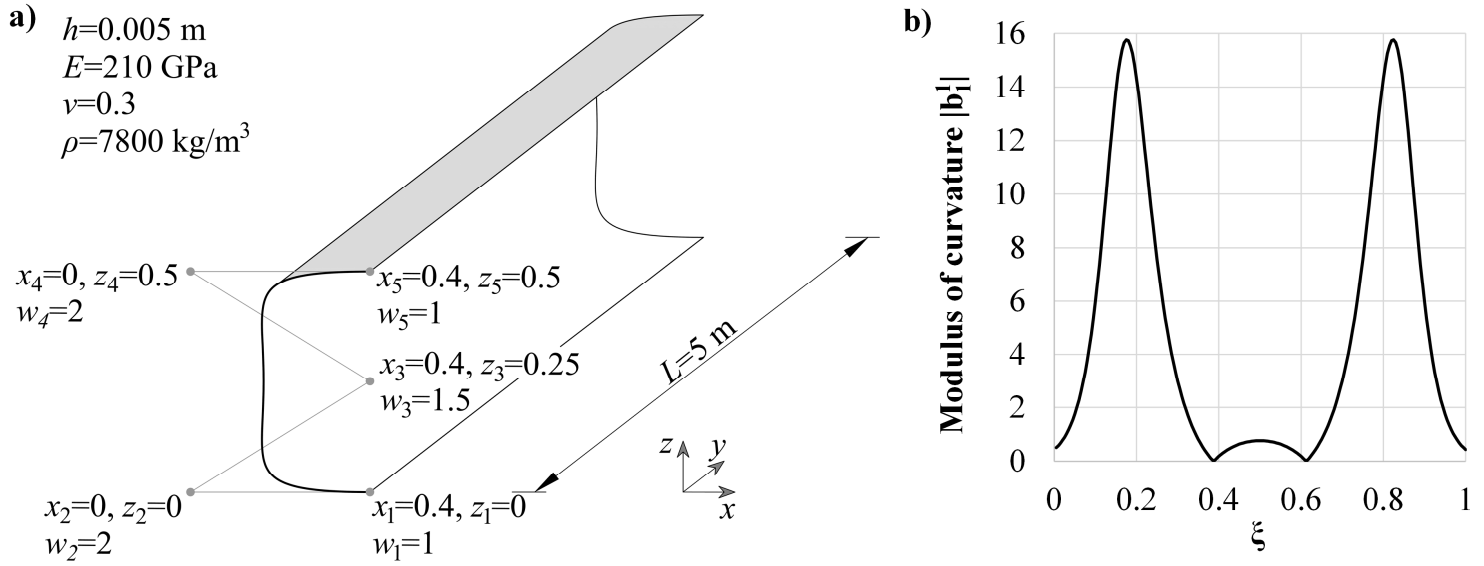

Fig. 20. The quartic cylinder. a) Geometry and material properties. The weights of control points are designated with $w_{i}$. b) distribution of the modulus of curvature along $\xi$ coordinate.

Table 3. The quartic cylinder. Comparison of the lowest eight eigenfrequencies [Hz].

RFD

\begin{tabular}{|c|c|c|c|c|c|c|}
\hline \multirow[b]{2}{*}{ Mode } & \multicolumn{2}{|c|}{ Abaqus STRI3 } & \multirow[b]{2}{*}{$\begin{array}{c}\text { Present } \\
80 \mathrm{~h}-4 \mathrm{p}-1 \mathrm{k}-4 \mathrm{t} \\
\left(\mathrm{n}_{\mathrm{DOF}}=2904\right) \\
\end{array}$} & \multicolumn{2}{|c|}{ Abaqus STRI3 } & \multirow[b]{2}{*}{$\begin{array}{c}\text { Present } \\
80 \mathrm{~h}-4 \mathrm{p}-1 \mathrm{k}-40 \mathrm{t} \\
\left(\mathrm{n}_{\mathrm{DOF}}=29040\right) \\
\end{array}$} \\
\hline & $\begin{array}{c}1608 \\
\text { elements } \\
\left(\mathrm{n}_{\mathrm{DOF}}=5304\right) \\
\end{array}$ & $\begin{array}{c}89000 \\
\text { elements } \\
\left(\mathrm{n}_{\mathrm{DOF}}=270540\right) \\
\end{array}$ & & $\begin{array}{c}1608 \\
\text { elements } \\
\left(\mathrm{n}_{\mathrm{DOF}}=5304\right) \\
\end{array}$ & $\begin{array}{c}89000 \\
\text { elements } \\
\left(\mathrm{n}_{\mathrm{DOF}}=270540\right) \\
\end{array}$ & \\
\hline 1 & 17.790 & 16.317 & 16.304 & 32.731 & 32.852 & 32.848 \\
\hline 2 & 25.832 & 24.301 & 24.270 & 35.971 & 33.561 & 33.584 \\
\hline 3 & 34.264 & 34.760 & 34.777 & 37.734 & 36.919 & 36.907 \\
\hline 4 & 35.720 & 35.638 & 35.627 & 47.258 & 44.007 & 43.961 \\
\hline 5 & 43.222 & 41.399 & 41.348 & 56.383 & 54.655 & 54.549 \\
\hline 6 & 50.702 & 49.455 & 49.481 & 58.940 & 55.766 & 55.781 \\
\hline 7 & 55.554 & 50.638 & 50.508 & 61.264 & 56.254 & 56.284 \\
\hline 8 & 61.402 & 61.480 & 61.478 & 64.056 & 63.712 & 63.714 \\
\hline
\end{tabular}




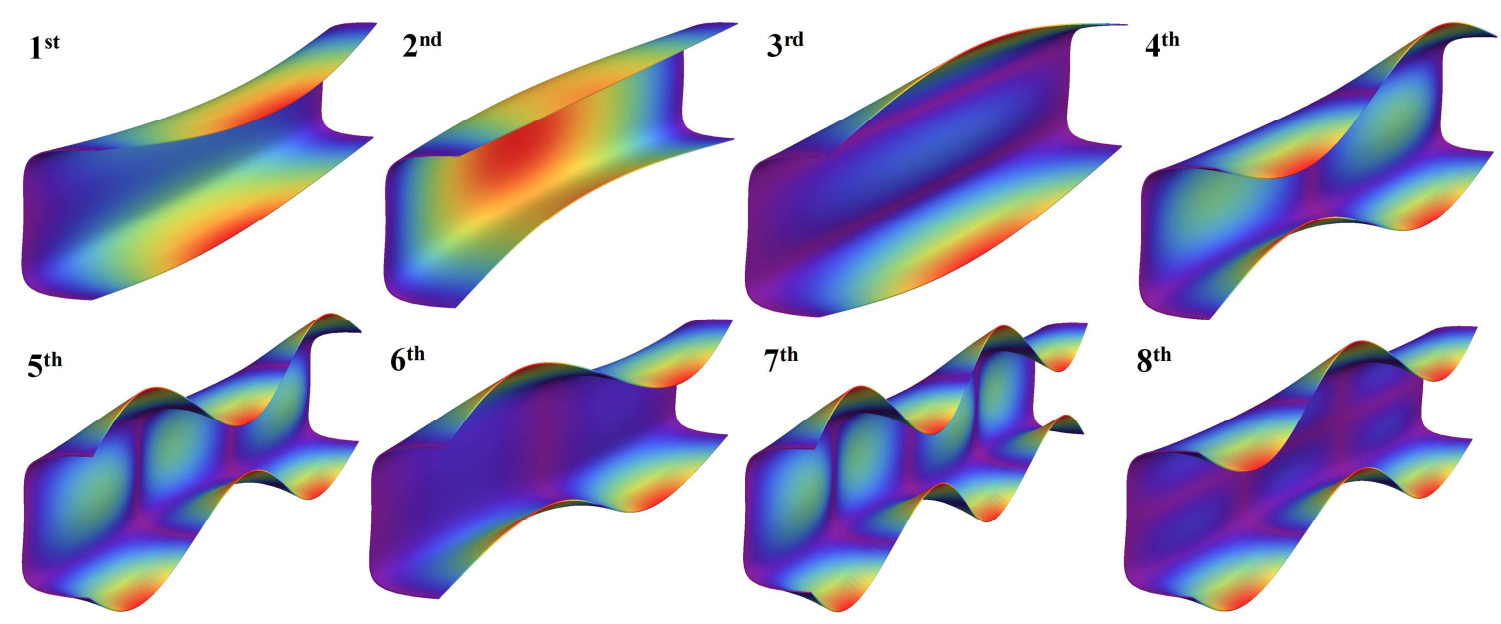

Fig. 21. The quartic cylinder with RFD supports. Mode shapes of the lowest eight modes.
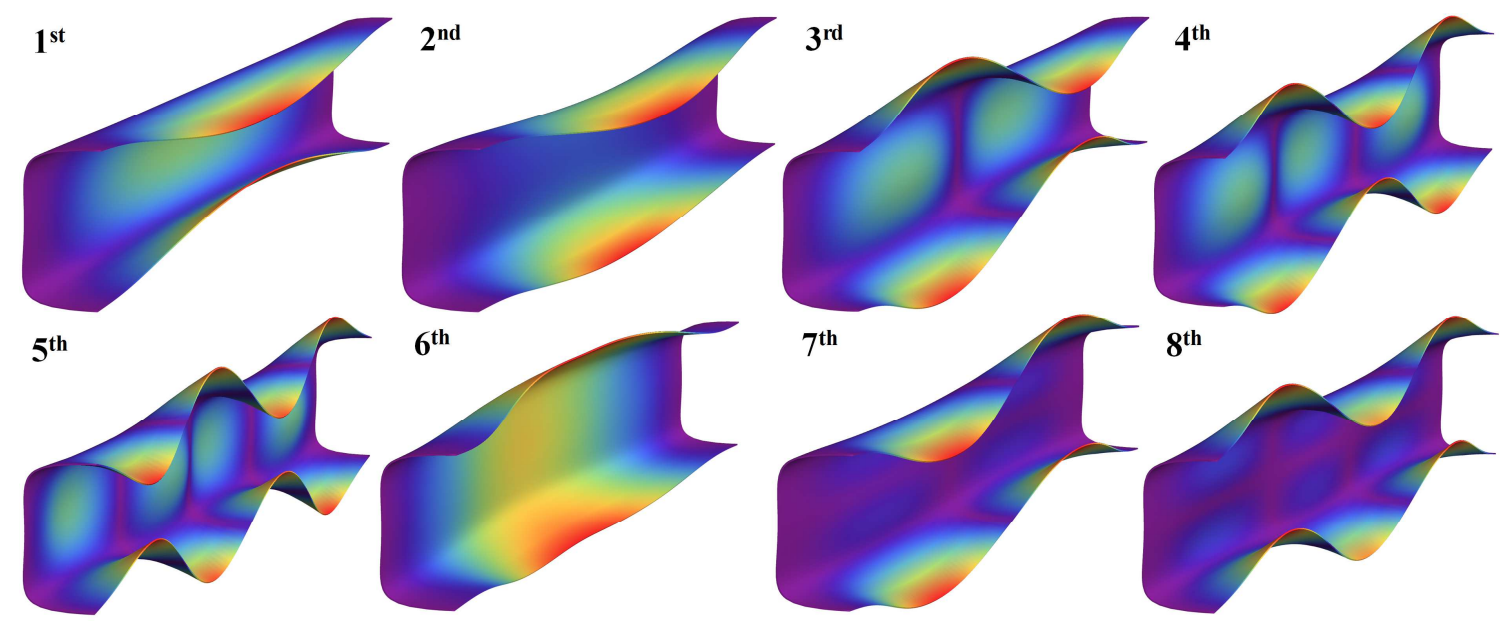

Fig. 22. The quartic cylinder with RRD supports. Mode shapes of the lowest eight modes.
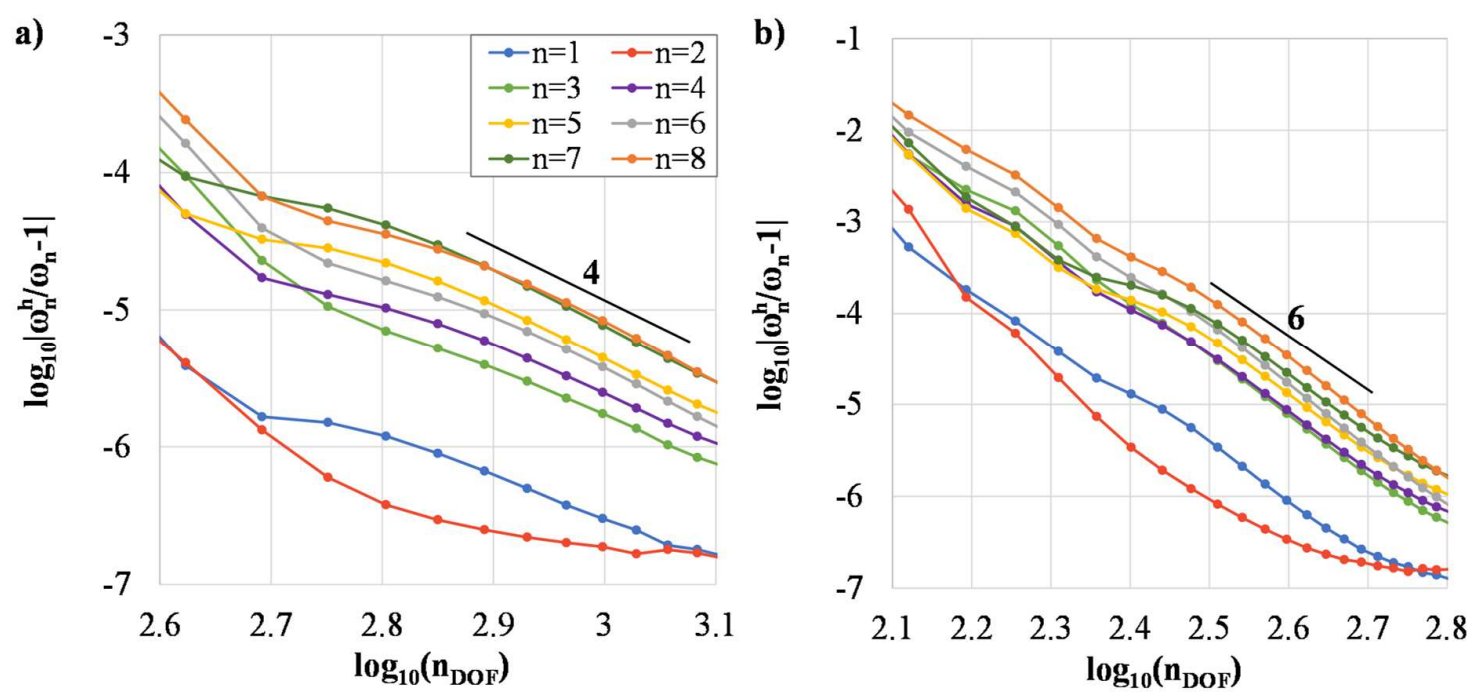

Fig. 23. The quartic cylinder with RFD supports. Convergence of the relative error for the lowest eight eigenfrequencies using the $h$-refinement: a) $m \mathrm{~h}-4 \mathrm{p}-1 \mathrm{k}-4 \mathrm{t}$; b) $m \mathrm{~h}-4 \mathrm{p}-3 \mathrm{k}-4 \mathrm{t}$. 

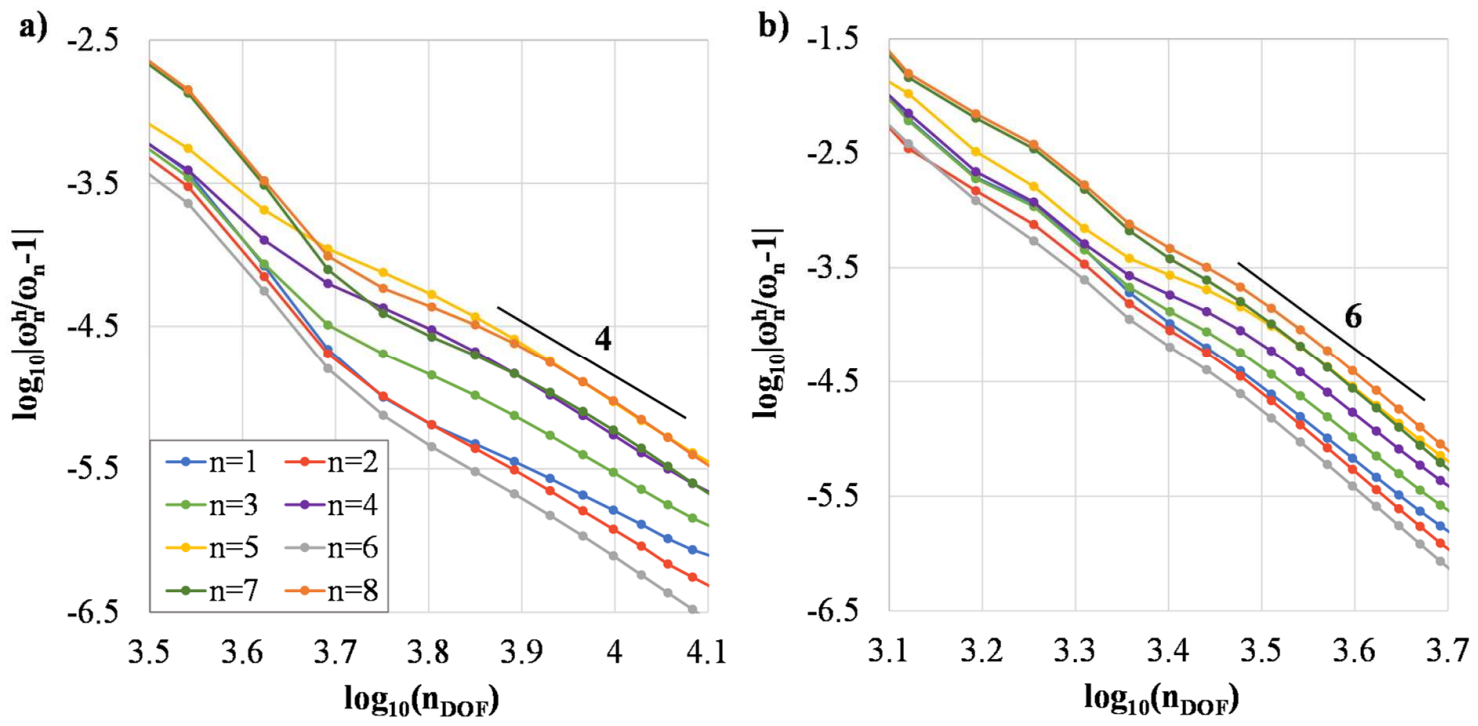

Fig. 24. The quartic cylinder with RRD supports. Convergence of the relative error for the lowest eight eigenfrequencies using the $h$-refinement: a) $m \mathrm{~h}-4 \mathrm{p}-1 \mathrm{k}-40 \mathrm{t}$; b) $m \mathrm{~h}-4 \mathrm{p}-3 \mathrm{k}-40 \mathrm{t}$.
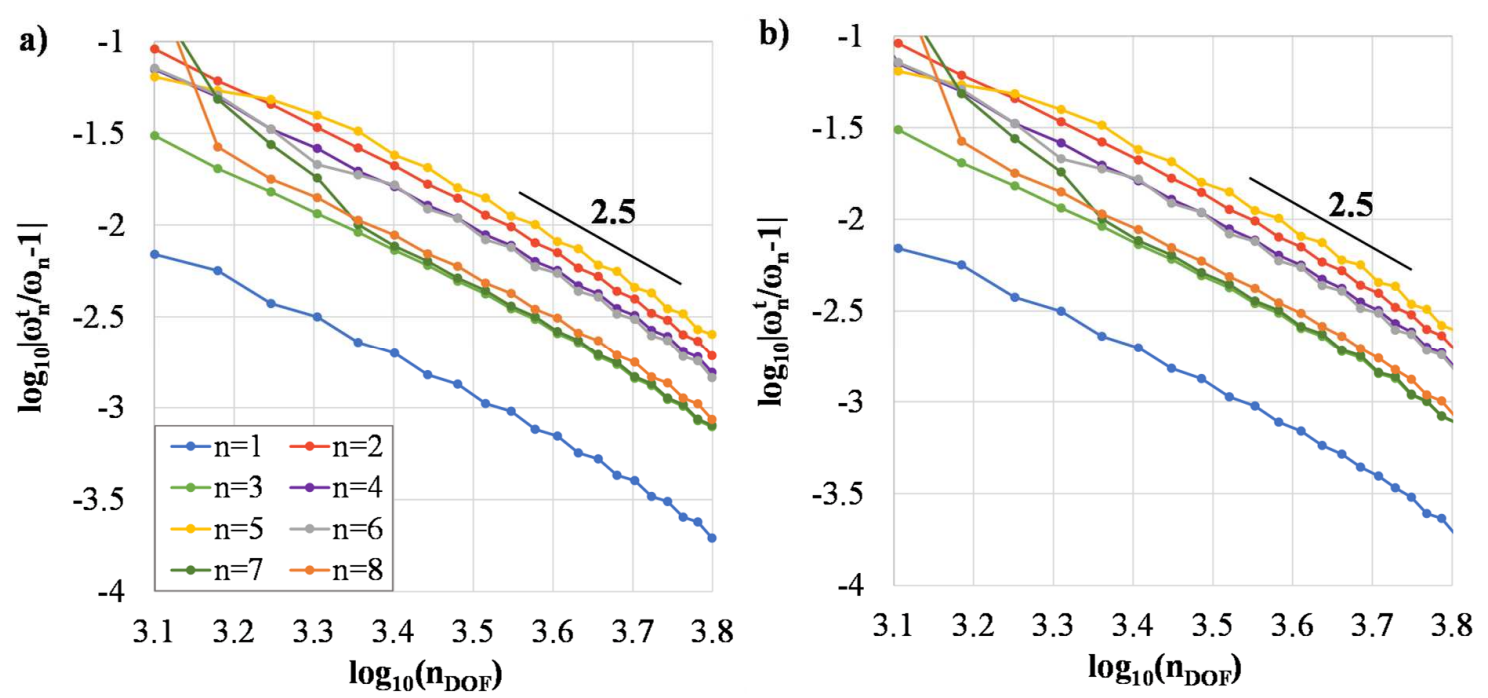

Fig. 25. The quartic cylinder with RRD supports. Convergence of the relative error for the lowest eight eigenfrequencies using the $t$-refinement: a) $80 \mathrm{~h}-4 \mathrm{p}-1 \mathrm{k}-j \mathrm{t}$; b) $80 \mathrm{~h}-4 \mathrm{p}-3 \mathrm{k}-j \mathrm{t}$.

\subsection{A cylinder with symmetric free-from cross section}

To emphasize the versatility of the presented technique, a structure with more involved cross-section is analyzed as a final example. The geometry is defined with eight quartic B-spline elements with $C^{3}$ interstrip continuity, Fig. 26a. The distribution of curvature in Fig. 26b depicts the complexity of adopted geometry. The maximum curviness of this shell is $K h \approx 0.096$.

The lowest eight frequencies for RRD and RFD boundary conditions are given in Table 4. As in the previous examples, an excellent agreement is observed between the FEA and FSIGA results. The mode shapes are very interesting and they are displayed in Fig. 27 and Fig. 28. Contrary to the previous examples, the maximum of two half-waves is present in all these modes, due to the increased shell length. Finally, the $h$-refinement tests are given in Fig. 29 for both the RRD and RFD shells. The orders of convergence are exceptional and similar to those obtained in the case of an open circular cylinder. 

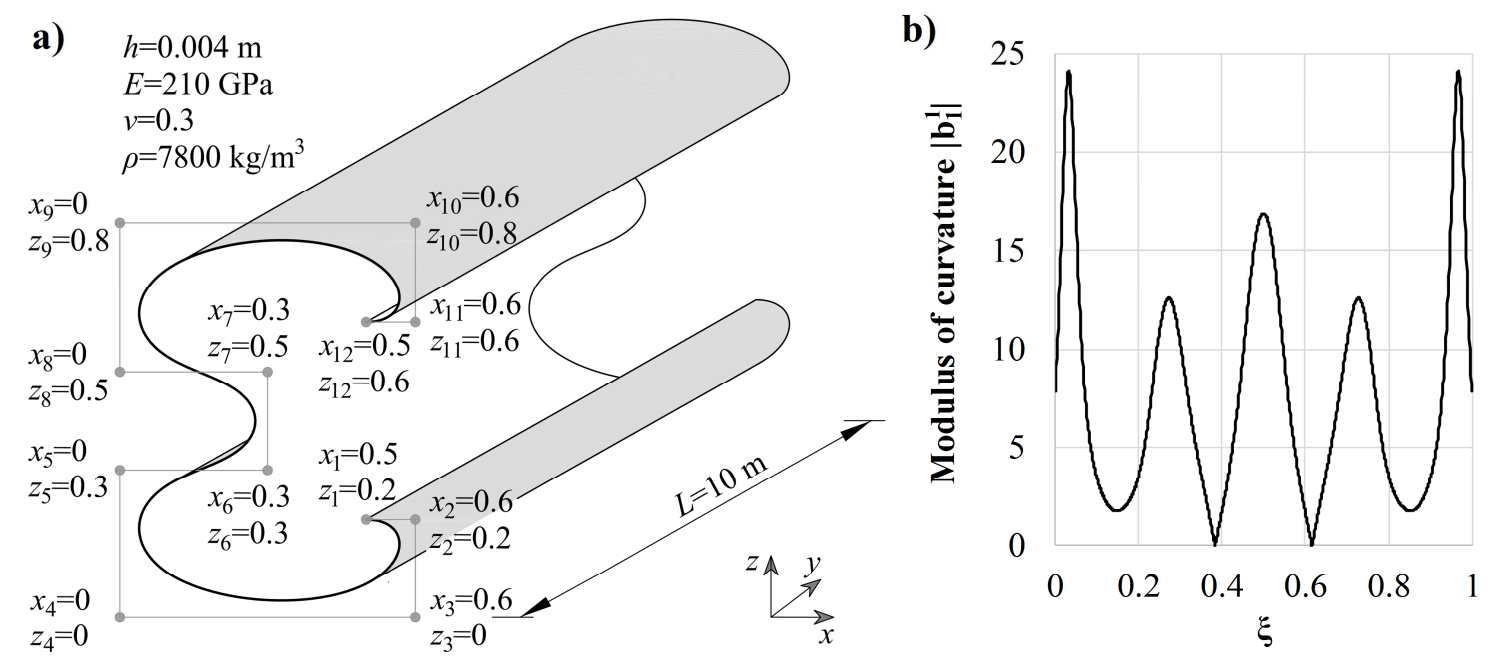

Fig. 26. The free-form cylinder. a) Geometry and material properties. b) Distribution of the modulus of curvature along $\xi$ coordinate.

Table 4. The free-form cylinder. Comparison of the lowest eight eigenfrequencies [Hz].

\begin{tabular}{|c|c|c|c|c|c|c|}
\hline \multirow{4}{*}{ Mode } & \multicolumn{3}{|c|}{ RFD } & \multicolumn{3}{|c|}{ RRD } \\
\hline & \multicolumn{2}{|c|}{ Abaqus STRI3 } & \multirow{2}{*}{$\begin{array}{c}\text { Present } \\
128 \mathrm{~h}-4 \mathrm{p}-3 \mathrm{k}- \\
5 \mathrm{t}\end{array}$} & \multicolumn{2}{|c|}{ Abaqus STRI3 } & \multirow{3}{*}{$\begin{array}{c}\text { Present } \\
128 \mathrm{~h}-4 \mathrm{p}-3 \mathrm{k}- \\
40 \mathrm{t} \\
(\mathrm{n} \mathrm{DOF}=15840)\end{array}$} \\
\hline & $\begin{array}{c}6000 \\
\text { elements }\end{array}$ & $\begin{array}{l}108000 \\
\text { elements }\end{array}$ & & $\begin{array}{l}6000 \\
\text { elements }\end{array}$ & $\begin{array}{c}108000 \\
\text { elements }\end{array}$ & \\
\hline & $\left(\mathrm{n}_{\mathrm{DOF}}=18786\right)$ & $(\mathrm{nDOF}=327654)$ & $\left(\mathrm{n}_{\mathrm{DOF}}=792\right)$ & $\left(\mathrm{n}_{\mathrm{DOF}}=18786\right)$ & $\left(\mathrm{nDOF}_{\mathrm{DOF}}=327654\right)$ & \\
\hline 1 & 7.0034 & 6.7446 & 6.7216 & 12.393 & 11.867 & 11.856 \\
\hline 2 & 8.5295 & 8.4002 & 8.3893 & 13.649 & 13.326 & 13.319 \\
\hline 3 & 14.187 & 13.920 & 13.915 & 25.142 & 24.476 & 24.490 \\
\hline 4 & 14.845 & 14.580 & 14.560 & 25.662 & 24.793 & 24.756 \\
\hline 5 & 20.253 & 19.395 & 19.334 & 30.334 & 29.044 & 29.027 \\
\hline 6 & 21.375 & 20.775 & 20.725 & 31.392 & 30.308 & 30.292 \\
\hline 7 & 29.087 & 29.168 & 29.159 & 34.021 & 33.296 & 33.224 \\
\hline 8 & 31.019 & 30.892 & 30.770 & 37.477 & 35.212 & 35.086 \\
\hline
\end{tabular}

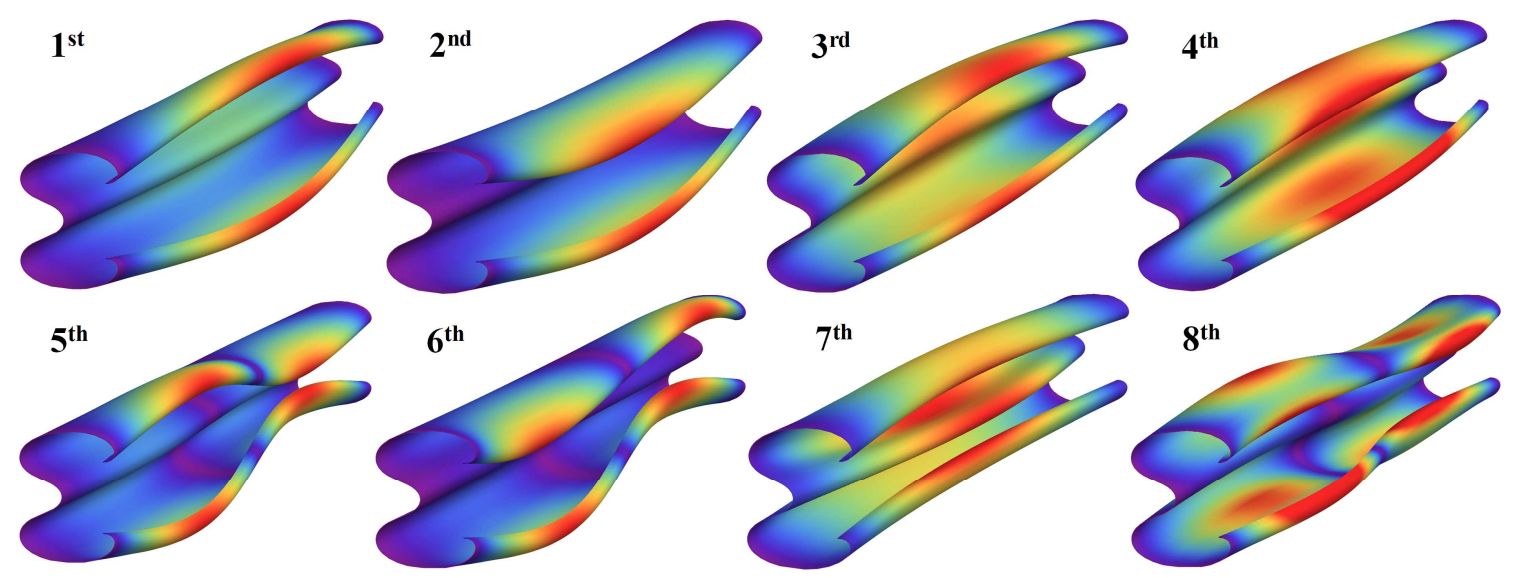

Fig. 27. The free-form cylinder. Mode shapes of the lowest eight modes. 


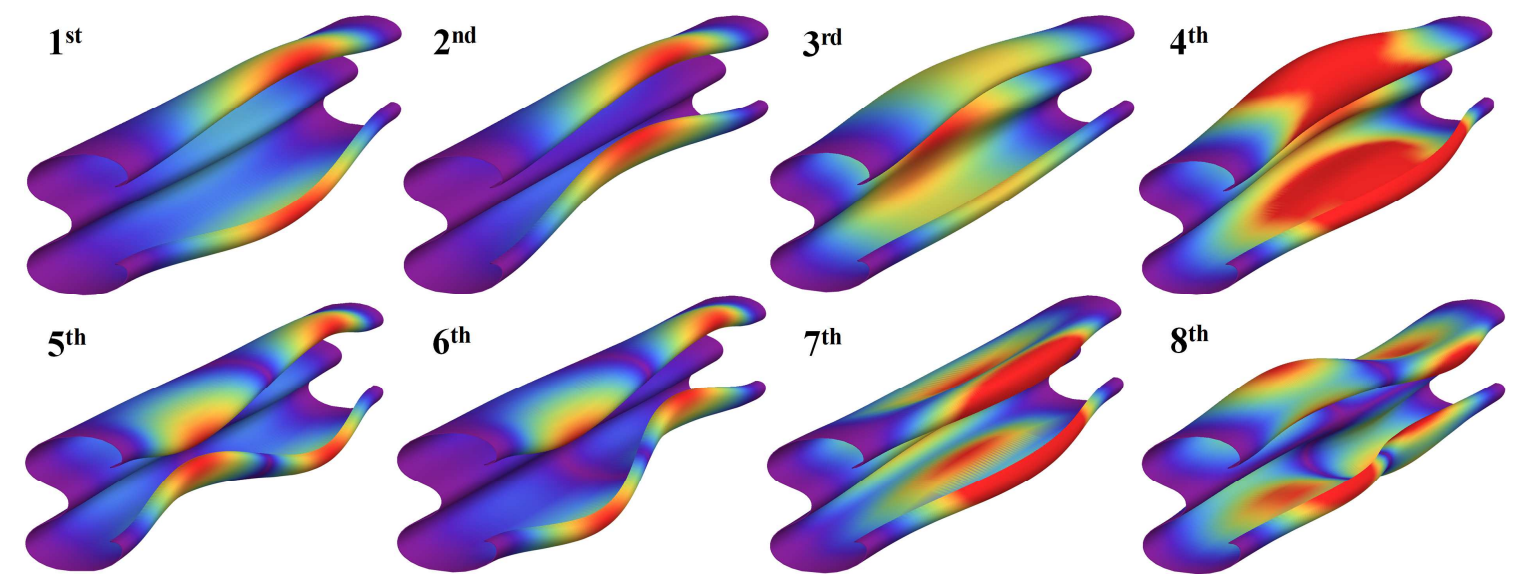

Fig. 28. The free-form cylinder. Mode shapes of the lowest eight modes.
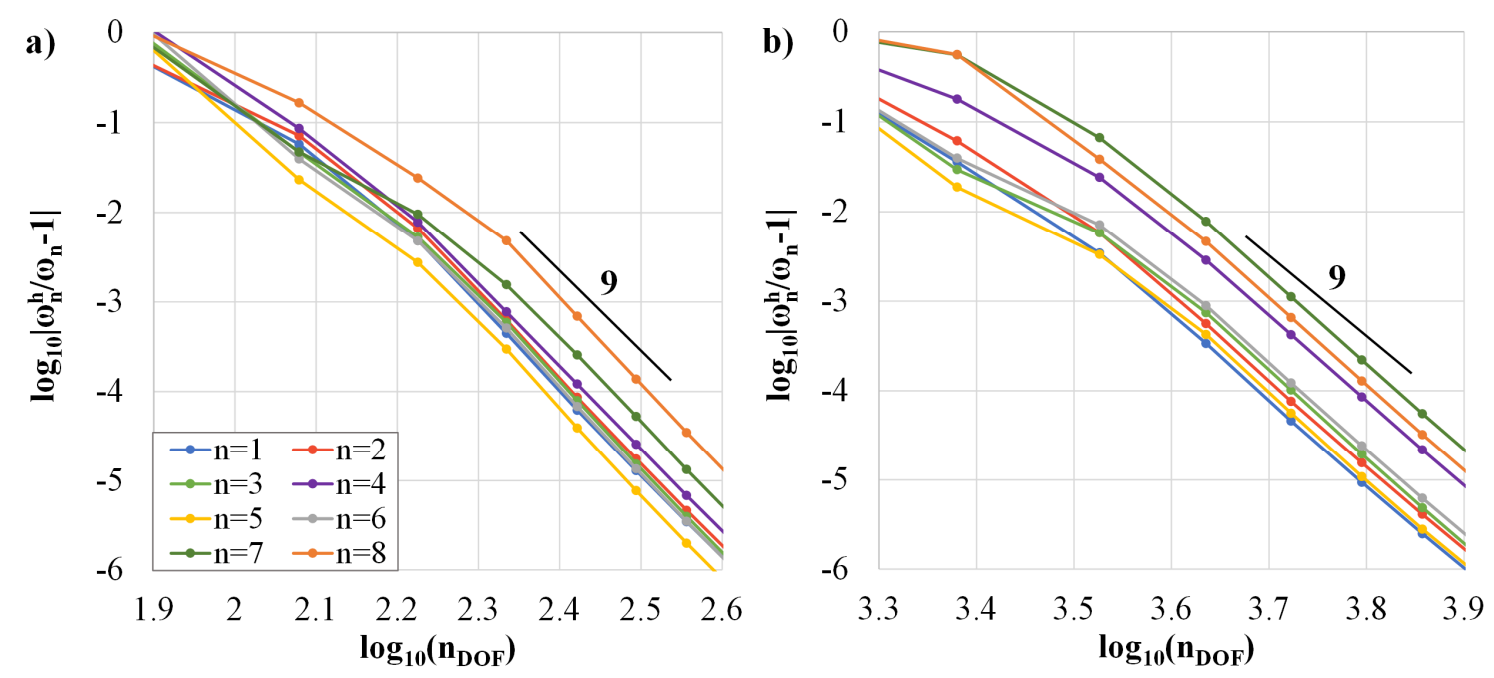

Fig. 29. The free-form cylinder. Convergence of the relative error for the lowest eight eigenfrequencies using the $h$-refinement with: a) RFD supports and $m \mathrm{~h}-4 \mathrm{p}-3 \mathrm{k}-2 \mathrm{t}$; b) RRD supports and $m \mathrm{~h}-4 \mathrm{p}-3 \mathrm{k}-40 \mathrm{t}$.

\section{Conclusions}

A novel IGFSM formulation is presented within the scope of the linear theory of thin shells. The method uses the IGA methodology for the discretization of 1D geometries and incorporates it into the semianalytical FSM. The isogeometric finite strip of a cylindrical shell is derived and implemented as a special case of a doubly curved strip. The method is verified and validated through detailed free vibration analysis of four characteristic examples. A key finding is that the derived method shares the features with the both underlying methods which results in the favorable convergence properties of $h p k t$-refined meshes.

The most important inherited property is increased continuity along both directions. This feature ameliorates the computational efficiency of FSIGA by improving the order of convergence and accuracy per DOF. Furthermore, for specific types of boundary conditions, the system equations decouple with respect to the series terms which allows for a remarkably effective analysis of such structures. Additional benefits come from the analytical integration of trigonometric functions and the exact description of arbitrarily curved reference geometry. These properties entail high accuracy and low computational cost of the proposed method, making it well-suited for the analysis of singly curved shells. 
The efficiency of the presented approach can be utilized for the optimization of cylindrical shells with respect to various parameters. For a more thorough conclusions regarding the capability of IGFSM to model arbitrarily curved shells, a full stress-strain analysis should be conducted with an emphasis on the nonlinear analysis. In addition, the method could be enriched so it could handle the multi-patch geometries, as well.

\section{Acknowledgments}

During this work, our beloved colleague and friend, Professor Gligor Radenković (1956-2019), passed away. The co-authors acknowledge that his unprecedented enthusiasm and love for the mechanics were crucial for much of our previous, present, and future researches. His students and colleagues will remember him by his incredibly infectious energy for both life and science.

This research is partially supported by the Ministry for Scientific-Technological Development, Higher Education and Information Society of Republic of Srpska through the project Dynamic stability of thin-walled structures using the Isogeometric finite strip method. This support is gratefully acknowledged.

We also acknowledge the support of the Austrian Science Fund (FWF): M 2806-N.

\section{Appendix A.}

Closed-form solutions of the eight integrals in Eq. (57) are given here. The solutions for six of them are the same for both type of considered supports:

$$
\begin{aligned}
& I_{1 m n}=\left\{\begin{array}{cc}
\frac{L}{2}, & m=n \\
0, & m \neq n
\end{array}, \quad I_{2 m n}=\left\{\begin{array}{cc}
-\frac{n^{2} \pi^{2}}{2 L}, & m=n \\
0, & m \neq n
\end{array}, \quad I_{3 m n}=\left\{\begin{array}{cc}
\frac{n^{4} \pi^{4}}{2 L^{3}}, & m=n \\
0, & m \neq n
\end{array}\right.\right.\right. \\
& I_{4 m n}=\left\{\begin{array}{cc}
\frac{n^{2} \pi^{2}}{2 L}, & m=n \\
0, & m \neq n
\end{array}, \quad I_{7 m n}=\left\{\begin{array}{cc}
\frac{n^{2} \pi^{2}}{2 L} & m=n \\
0 & m \neq n
\end{array}, \quad I_{8 m n}=\left\{\begin{array}{cc}
\frac{L}{2} & m=n . \\
0 & m \neq n
\end{array}\right.\right.\right.
\end{aligned}
$$

The remaining two integrals are:

$$
I_{5 m n}=\left\{\begin{array}{cc}
-\frac{n \pi}{2}, & m=n \\
0, & m \neq n
\end{array}, \quad I_{6 m n}=\left\{\begin{array}{cc}
\frac{n \pi}{2}, & m=n \\
0, & m \neq n
\end{array}\right.\right.
$$

for RFD supports, and:

$$
I_{5 m n}=\left\{\begin{array}{cc}
0 & m=n \\
\frac{\left[(-1)^{m+n}-1\right] m n}{m^{2}-n^{2}} & m \neq n
\end{array}, \quad I_{6 m n}=\left\{\begin{array}{cc}
0 & m=n \\
\frac{\left[(-1)^{m+n}-1\right] m n}{n^{2}-m^{2}} & m \neq n
\end{array} .\right.\right.
$$

for RRD supports. Evidently, these two integrals couple the system equations for the case of RRD supports.

\section{Literature}

[1] P. Naghdi, "The Theory of Shells and Plates," in Truesdell C. (eds) Linear Theories of Elasticity and Thermoelasticity, Berlin, Heidelberg, Springer, 1973, pp. 425-640.

[2] A. Leissa, Vibration of Shells, Washington, DC: Scientific and Technical Information Office, National Aeronautics and Space Administration, 1973. 
[3] W. Soedel, Vibrations of Shells and Plates, New York: Marcel Dekker Inc., 2004.

[4] M. Qatu, Vibration of Laminated Shells and Plates, Elsevier Academic Press, 2004.

[5] T. Ye, G. Jin, Y. Chen and S. Shi, "A unified formulation for vibration analysis of open shells with arbitrary boundary conditions," International Journal of Mechanical Sciences, vol. 81, pp. 42-59, 2014.

[6] K. Forsberg, "Influence of Boundary Conditions on the Modal Characteristics of Thin Cylindrical Shells," AIAA Journal, vol. 2, no. 12, pp. 2150-2157, 1964.

[7] G. Warburton, "Vibration of Thin Cylindrical Shells," Journal of Mechanical Engineering Science, vol. 7, no. 4, pp. 399-407, 1965.

[8] L. Dai, T. Yang, J. Du, W. Li and M. Brennan, "An exact series solution for the vibration analysis of cylindrical shells," Applied Acoustics, vol. 74, pp. 440-449, 2013.

[9] M. Cammalleri and A. Constanza, "A closed-form solution for natural frequencies of thin-walled cylinders with clamped edges," International Journal of Mechanical Sciences, vol. 110, pp. 116126, 2016.

[10] S. Yu, W. Cleghorn and R. Fenton, "On the accurate analysis of free vibration of open circular cylindrical shells," Journal of Sound and Vibration, vol. 188, no. 3, pp. 315-336, 1995.

[11] A. Selmane and A. Lakis, "Dynamic analysis of anisotropic open cylindrical shells," Computers \& Structures, vol. 62, no. 1, pp. 1-12, 1997.

[12] D. Shi, Y. Zhao, Q. Wang, X. Teng and F. Pang, "A Unified Spectro-Geometric-Ritz Method for Vibration Analysis of Open and Closed Shells with Arbitrary Boundary Conditions," Shock and Vibration, vol. 2016, pp. 1-30, 2016.

[13] Y. Xing, B. Liu and T. Xu, "Exact solutions for free vibration of circular cylindrical shells with classical boundary conditions," International Journal of Mechanical Sciences, vol. 75, pp. 178188, 2013.

[14] G. Yamada, T. Irie and Y. Tagawa, "Free vibration of non-circular cylindrical shells with variable circumferential profile," Journal of Sound and Vibration, vol. 95, no. 1, pp. 117-126, 1984.

[15] A. Grigorenko, S. Puzyrev and E. Volchek, "Investigation of free vibrations of noncircular cylindrical shells by the spline-collocation method," Journal of Mathematical Sciences , vol. 185, p. 824-836, 2012.

[16] Y. Cheung, "The finite strip method in the analysis of elastic plates with two opposite simply supported ends," in Proceedings - Institution of Civil Engineers, 1968.

[17] T. Hughes, The Finite Element Method: Linear Static and Dynamic Finite Element Analysis, New Jersey: Prentice-Hall, 1987.

[18] Y. Cheung and L. Tham, The finite strip method, CRC Press, 1997.

[19] Y. Loo and A. Cusens, The finite strip method in bridge engineering, Viewpoint publications, 1978. 
[20] D. D. Milašinović, The Finite Strip Method in Computational Mechanics, Faculties of Civil Engineering: University of Novi Sad, Technical University of Budapest and University of Belgrade: Subotica, Budapest, Belgrade, 1997.

[21] A. Borković, S. Kovačević, D. Milašinović, G. Radenković, O. Mijatović and V. GolubovićBugarski, "Geometric nonlinear analysis of prismatic shells using the semi-analytical finite strip method," Thin-Walled Structures, vol. 117, pp. 63-88, 2017.

[22] A. Borković, N. Mrđa and S. Kovačević, "Dynamical analysis of stiffened plates using the compound strip method," Engineering Structures, vol. 50, pp. 56-67, 2013.

[23] Y. Cheung, W. Li and L. Tham, "Free vibration analysis of singly curved shell by spline finite strip method," Journal of Sound and Vibration, vol. 128, no. 3, pp. 411-422, 1989.

[24] W. Li, L. Tham, Y. Cheung and S. Fan, "Free vibration analysis of doubly curved shells by spline finite strip method," Journal of Sound and Vibration, vol. 140, no. 1, pp. 39-53, 1990.

[25] F. Au and Y. Cheung, "Free vibration and stability analysis of shells by the isoparametric spline finite strip method," Thin-Walled Structures, vol. 24, no. 1, pp. 53-82, 1996.

[26] T. Hughes, J. Cottrell and Y. Bazilevs, "Isogeometric analysis: CAD, finite elements, NURBS, exact geometry and mesh refinement," Computer methods in applied mechanics and engineering, vol. 194, pp. 4135-4195, 2005.

[27] L. Beirão da Veiga, A. Buffa, J. Rivas and G. Sangalli, "Some estimates for h-p-k-refinement in Isogeometric Analysis," Numer. Math., vol. 118, no. 2, pp. 271-305, June 2011.

[28] G. Radenković and A. Borković, "Linear static isogeometric analysis of an arbitrarily curved spatial Bernoulli-Euler beam," Computer Methods in Applied Mechanics and Engineering, vol. 341, pp. 360-396, 2018.

[29] J. Cottrell, A. Reali, Y. Bazilevs and T. Hughes, "Isogeometric analysis of structural vibrations," Computer Methods in Applied Mechanics and Engineering, vol. 195, no. 41-43, pp. 5257-5296, 2006.

[30] A. Borković, S. Kovačević, G. Radenković, S. Milovanović and D. Majstorović, "Rotation-free isogeometric dynamic analysis of an arbitrarily curved plane Bernoulli-Euler beam," Engineering Structures, vol. 181, pp. 192-215, 2019.

[31] J. Kiendl, K.-U. Bletzinger, J. Linhard and R. Wüchner, "Isogeometric shell analysis with Kirchhoff-Love elements," Computer Methods in Applied Mechanics and Engineering, vol. 198, no. 49-52, pp. 3902-3914, 2009.

[32] G. Radenković, A. Borković and B. Marussig, Nonlinear static isogeometric analysis of arbitrarily curved Kirchhoff-Love shells, 2020.

[33] N. Nguyen-Thanh, W. Li and K. Zhou, "Static and free-vibration analyses of cracks in thin-shell structures based on an isogeometric-meshfree coupling approach," Computational Mechanics, vol. 62, p. pages1287-1309, 2018.

[34] P. Tan, N. Nguyen-Thanh, T. Rabczuk and K. Zhou, "Static, dynamic and buckling analyses of 3D FGM plates and shells via an isogeometric-meshfree coupling approach," Composite Structures, vol. 198, p. 2018, 2018. 
[35] M. Shahmohammadi, M. Azhari, M. Mehdi, M. Saadatpour and S. Sarrami-Foroushani, "Geometrically nonlinear analysis of sandwich FGM and laminated composite degenerated shells using the isogeometric finite strip method," Computer Methods in Applied Mechanics and Engineering, vol. 371, p. 113311, 2020.

[36] A. Borković, S. Kovačević, G. Radenković, S. Milovanović and M. Guzijan-Dilber, "Rotationfree isogeometric analysis of an arbitrarily curved plane Bernoulli-Euler beam," Computer Methods in Applied Mechanics and Engineering, vol. 334, pp. 238-267, 2018.

[37] V. Kumar and A. Singh, "Vibration Analysis Of Non-circular Cylindrical Shells Using Bezier Functions," Journal of Sound and Vibration, vol. 161, no. 2, pp. 333-354, 1993.

[38] G. Radenković, Finite rotation and finite strain isogeometric structural analysis (in Serbian), Belgrade: Faculty of Architecture, 2017.

[39] L. Piegl and W. Tiller, The NURBS Book, Springer, 1995.

[40] M. Bischoff, K. Bletzinger, W. Wall and E. Ramm, "Models and Finite Elements for Thin-Walled Structures," in Encyclopedia of Computational Mechanics, 2004.

[41] J. Kiendl, M.-C. Hsu, M. Wu and A. Reali, "Isogeometric Kirchhoff-Love shell formulations for general hyperelastic materials," Computer Methods in Applied Mechanics and Engineering, vol. 291, pp. 280-303, 2015.

[42] T. Hughes, A. Reali and G. Sangalli, "Efficient quadrature for NURBS-based isogeometric analysis," Computer Methods in Applied Mechanics and Engineering, vol. 100, no. 5-8, pp. 301313, January 2010.

[43] F. Calabro, G. Sangalli and M. Tani, "Fast formation of isogeometric Galerkin matrices by weighted quadrature," Comput. Methods Appl. Mech. Engrg., vol. 316, p. 606-622, 2017.

[44] Abaqus Documentation, Dassault Systèmes, 2014.

[45] K. Heki, "Vibrations of cylindrical shells," Journal of the Institute of Polytechniques, Osaka City University, Series F, vol. 1, pp. 1-36, 1957.

[46] Z. Li, J. Abreu, J. Leng, S. Adany and B. Schafer, "Review: Constrained finite strip method developments and applications in cold-formed steel design," Thin-Walled Structures, vol. 81, pp. 2-18, 2014. 